\title{
Review of Soluble Biomarkers of Osteoarthritis: Lessons From Animal Models
}

\author{
Catherine B. Legrand', Cécile J. Lambert', Fanny V. Comblain', \\ Christelle Sanchez', and Yves E. Henrotin ${ }^{1,2}$
}

\begin{abstract}
Objective. Osteoarthritis (OA) is one of the leading causes of disability within the adult population. Currently, its diagnosis is mainly based on clinical examination and standard radiography. To date, there is no way to detect the disease at a molecular level, before the appearance of structural changes and symptoms. So an attractive alternative for monitoring OA is the measurement of biochemical markers in blood, urine, or synovial fluid, which could reflect metabolic changes in joint tissue and therefore disease onset and progression. Animal models are relevant to investigate the early stage of OA and metabolic changes occurring in joint tissues. The goal of this narrative review is to summarize the scientific data available in the literature on soluble biomarkers in animal models of OA. Design. A literature search was conducted using the PubMed/ Medline and Scopus databases between February 1995 and December 2015. All original articles, systematic and narrative reviews published in French or in English were considered. Results. We summarized the data of 69 studies and proposed a classification scheme for OA biomarkers in animal studies, largely inspired by the BIPEDS classification. Conclusions. Studies about biomarkers and animal models indicate that some markers could be valuable to monitor OA progression and assess therapeutic response in some animal models.
\end{abstract}

\section{Keywords}

osteoarthritis, diagnosis, animal models, biomarkers, diagnostics, BIPED classification

\section{Introduction}

Osteoarthritis (OA), a disease of the entire joint, is characterized by, synovial inflammation, ligament hyperlaxity, and abnormal subchondral bone remodeling. Currently, its diagnosis is mainly based on radiographic criteria (e.g., joint space width) and clinical symptoms (e.g., pain and loss of function). ${ }^{1,2}$ However, a diagnosis of the disease at a preradiographic stage, when the first molecular changes occur in joint tissues, would be helpful for a better management of the disease. In this purpose, imaging techniques such as ultrasound and magnetic resonance imaging (MRI) have been developed, but their use is limited by their cost and availability. An alternative is the measurement of soluble biomarkers in biological fluids, not only to early diagnose but also to predict the progression of the disease and to assess the effectiveness of treatments. ${ }^{2}$ Furthermore, soluble biomarkers can be used as "drug development tools" at preclinical stages and throughout clinical development in phase I to IV trials. ${ }^{3}$ At the preclinical phases, the 3 major applications of biomarkers are their use in toxicology studies, in the selection of appropriate animal models and lead compounds and in the identification of the mechanisms of action of an intervention.

In 2006, the BIPED classification was created with the aim of providing a common framework for communication in the field. ${ }^{1}$ The acronym "BIPED" stands for Burden of disease, Investigate, Prognostic, Efficacy of intervention, and Diagnostic. The "BIPED" classification was subsequently revised to "BIPEDS," including "Safety." Many reviews on biochemical markers using the BIPEDS classification have been published. ${ }^{2,4-6}$ In those reviews, biomarkers have been classified based on data coming from human clinical trials. These soluble biomarkers have never been

\footnotetext{
'Bone and Cartilage Research Unit, Arthropôle Liège, University of Liège, Institute of Pathology, CHU Sart-Tilman, Liège, Belgium

2Department of Physical Therapy and Rehabilitation, Princess Paola Hospital, Vivalia, Marche-en-Famenne, Belgium

Corresponding Author:

Yves E. Henrotin, Bone and Cartilage Research Unit, Arthropôle Liège, University of Liège, Institute of Pathology, Level 5, CHU Sart Tilman, 4000 Liège, Belgium.

Email:yhenrotin@ulg.ac.be
} 
classified based on information coming from animal studies, although animal models are valuable to validate biomarkers.

Currently, several animal models of OA in rabbits, dogs, rats, mice, guinea pigs, horses are conventionally used, mainly to test therapeutic modalities. Three general categories exist for in vivo OA models: spontaneously occurring OA (including genetically modified animals), mechanically induced accelerated OA by surgery- (e.g., anterior cruciate ligament transection [ACLT]), trauma-, or fracture-induced posttraumatic $\mathrm{OA},{ }^{7,8}$ and chemically induced $\mathrm{OA}$ by intraarticular injection of chondrotoxic (e.g., iodoacetic acid), pro-inflammatory (e.g., interleukin-1 $\beta[\mathrm{IL}-1 \beta]$ ), or catabolic (e.g., matrix metalloproteinase [MMP]) substances. ${ }^{9-11}$

The goal of this narrative review was to synthesize scientific literature on soluble biomarkers measured in biological fluids in different animal models of OA and to classify these biomarkers according the BIPEDS classification.

\section{Methods}

The literature search was performed using the PubMed/ Medline and Scopus databases between February 1995 and December 2015. Searches were performed using the search terms "biomarkers," "osteoarthritis," and "animal model." The following word association was used in Medline: ["Biomarkers" OR "Markers"] AND ["Osteoarthritis"] AND ["Animal model"]. In the Scopus database, the code TITLE-ABS-KEY, a combined field that searches article titles, abstracts, and keywords was used. The following words association was used: biomarkers and osteoarthritis and animal model. All original papers, systematic and narrative reviews published in French or in English between February 1995 and December 2015 were included.

The first author (C.B.L.) selected the articles on the basis of the title and abstract and then assessed whether the study met the inclusion criteria. Then the full article was retrieved. The flow diagram for selection of studies is presented in Figure 1. Data are shown according to the species. By this way, the knowledge on biomarkers in a particular model is summarized. This approach was considered as useful for researchers using that particular model. Thereafter, we propose a classification of these biomarkers according their ability to predict the progression of the disease, to monitor the effect of a treatment or to assess the severity of OA lesions. The classification was performed based on the following definitions:

- Burden of disease (B): Biochemical marker concentrations correlated statistically and significantly with parameters of disease severity (e.g., histological, macroscopic, imaging score)

- Investigative (I): Biochemical marker concentrations that have not met criteria for another category yet

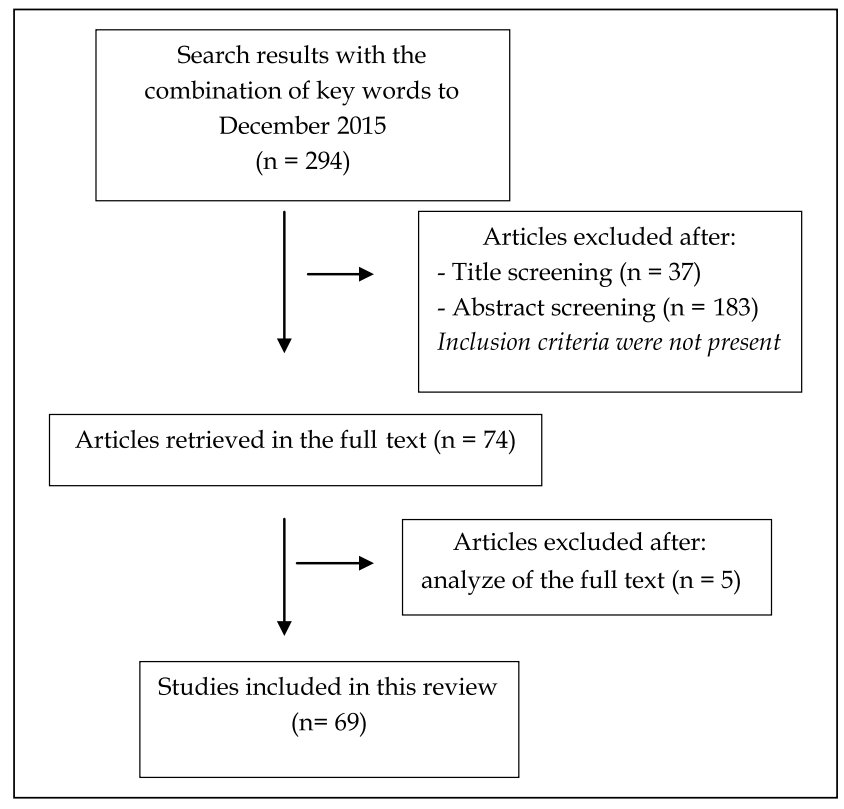

Figure I. Flow diagram for selection of study.

- Prognostic (P): Biochemical marker concentrations correlated significantly with the progression of disease severity parameters

- Efficacy of intervention (E): Biochemical marker concentrations differed statistically and significantly between animals with or without treatment, or before and after treatment with animals

- Diagnostic (D): Biomarker concentrations were statistically significantly different between animals that developed the disease and those that did not

- Safety (S): Biomarker able to reflect tissue and or organ toxicity of an agent or intervention

\section{Results}

\section{Rabbits}

In rabbits, a variety of surgical procedures on menisci and ligaments were described to induce knee joint laxity, and subsequently abnormal mechanical strain. These surgical procedures included cruciate ligament transection, with or without medial and collateral ligament section, total or partial meniscectomy, and creation of bucket-handled tears of the meniscus.

Prostaglandin (PG) E2 and C-reactive protein (CRP) serum levels were significantly increased 6 weeks after ACLT. $^{12}$ In contrast, no significant differences between the pre-ACLT and the post-ACLT values of serum keratan sulfate (KS), hyaluronic acid (HA), and chondroitin sulfate (CS) 846 epitope were observed. ${ }^{13}$ However, a significant correlation between histological score and post-ACLT HA concentration was found for the medial femoral condyle. In 
contrast, thrombin-cleaved osteopontin (OPN) levels in synovial fluid progressively and significantly increased after posterior cruciate ligament transection (PCLT) to reach a maximum after 4 weeks. ${ }^{14}$ A positive significant correlation was found between thrombin-cleaved OPN synovial fluid levels and the macroscopic and histological scores. ${ }^{14}$ In a longitudinal study, Duclos et al. ${ }^{15}$ followed the evolution of serum C-telopeptide of type II collagen (CTX-II) concentration in adult (10-month-old) and growing animals (2-month-old) after ACLT. CTX-II was significantly higher in ACLT-adult rabbits than nonoperated adult animals, while in young animals CTX-II was similar in both groups. In adult ACLT-rabbits, the CTX-II evolution showed 2 distinct elevations, one during the 3 first weeks after surgery, followed by a decreased until the fourth week, and a second peak at the twelfth week. In young animals, CTX-II levels increased rapidly and reached a maximum at week 2 and then, decreased over time until the week $20 .{ }^{15} \mathrm{In}$ experimental $\mathrm{OA}$ induced by anterior and posterior cruciate ligaments transection and medial meniscus excision, Zuo et al. ${ }^{16}$ investigated the evolution of serum bone morphogenetic protein-2 (BMP-2), CTX-II, and cartilage oligomeric protein (COMP). The BMP-2 levels were increased at various times after surgery, and significant differences were observed between the OA and the control groups. CTX-II levels were significantly elevated at different intervals after surgery. Significant differences in the COMP levels from W2 to W20 were observed between the OA and the control groups. ${ }^{16}$

Another research observed a significant increase of CTX-II in synovial fluid in rabbit with medial meniscectomy. ${ }^{17}$ Moreover, highly significant correlations between synovial fluid CTX-II levels and the macroscopic and microscopic scoring results were found.

\section{Rats}

In rats, a variety of experimental OA model exist. The most commonly used rat OA model is the surgically induced medial meniscal tear (MMT), ACLT alone or in combination with (partial) medial meniscectomy (ACLT + pMMx) ${ }^{18}$ Intra-articular monoiodoacetate (MIA) injection is also frequently used. Ovariectomy in rats was proposed to mimic postmenopausal OA in humans. ${ }^{19}$

In ACLT model, serum level of C2C, a biomarker of type II collagen degradation, was decreased by moderate running on a motorized treadmill (at a speed of $18 \mathrm{~m} / \mathrm{min}$ for 30 $\mathrm{min} / \mathrm{d}, 3$ days per week) while the serum level of CPII, a biomarker of type II synthesis, was decreased by intense running (at a speed of $18 \mathrm{~m} / \mathrm{min}$ for $60 \mathrm{~min} / \mathrm{d}, 3 \mathrm{~d} / \mathrm{wk}$ ) 2 and 4 weeks after surgery. ${ }^{20}$ In addition, in all the groups at 2 and 4 weeks after surgery, the Osteoarthritis Research Society International (OARSI) histological score was slightly but significantly correlated positively with the $\mathrm{C} 2 \mathrm{C} /$
CPII ratios. ${ }^{15}$ In ACLT rats, serum CTX-II and COMP levels were increased and only CTX-II correlated with the modified Mankin score. ${ }^{21}$

In ACLT and MMX rat model, low-intensity pulsed ultrasound (LIPUS) does not affect the degradation of type II collagen at 7 and 14 days after the operation. In contrast, after 28 days, CPII was significantly increased in LIPUS group compared to control group. ${ }^{22}$

The oral administration of high dose of glucosamine hydrochloride $(1000 \mathrm{mg} / \mathrm{kg} / \mathrm{d}$ for 56 days) prevented the increase of CTX-II serum levels ${ }^{23}$ and high dose of alendronate (ALN), a potent inhibitor of osteoclastic bone resorption, significantly decreased serum levels of COMP in rats with ACLT-induced OA. ${ }^{24}$ Another study investigated the effects of physical exercise in healthy young, adult, and aged rats on the expression of lubricin. As expected, the results showed that lubricin levels in rat synovial fluid were decreased with aging. ${ }^{25}$

In ACLT/pMMX rat model, treatment of OA rat knees with pharmacologic inhibitors of transforming growth factor- $\alpha$ (TGF- $\alpha$ ) (AG1478) and CCL2 (RS504393) signaling pathway, significantly increased serum CPII levels but only AG1478 significantly reduced serum C2C levels and the $\mathrm{C} 2 \mathrm{C} / \mathrm{CPII}$ ratio compared with untreated $\mathrm{OA}$ rats. This finding suggested that TGF- $\alpha / C C L 2$ is an important promoter of articular cartilage degradation in posttraumatic OA. ${ }^{26}$ In another study, oral administration of a high dose of BIS076 $(245 \mathrm{mg} / \mathrm{kg})$, a natural porcine cartilage extract associated with hydroxyapatite and vitamin $\mathrm{D}_{3}$, reduced serum levels of CTX-II and COMP, as well as MMP-3 in OVX and ACLT rats. ${ }^{27}$

In ovariectomized (OVX) rats, serum COMP level was decreased by a combination of estrogen and progesterone hormones 10 weeks after OVX surgery. ${ }^{28}$ In another study, CTX-II was also increased in the OVX rats compared with the sham-operated group. ${ }^{19}$

Several inflammatory mediators were investigated in MIA-induced OA in rats. Orita et al..$^{29}$ showed a significant increase in the concentrations of tumor necrosis factor- $\alpha$ (TNF- $\alpha$ ) and interleukine-6 (IL-6) starting on day 1 after the injection which reached a maximum at day 4 , and thereafter, gradually decreased as the post injection period progressed. The concentration of nerve growth factor (NGF) was significantly increased 1 week after the injection. ${ }^{29}$ In 2010, Swearingen et al.$^{30}$ showed a time-dependent increase in the release of aggrecanase-cleaved aggrecan (NITEGE and ARGS neoepitopes) into the synovial fluid of the MIA OA knee joints.

These results were consistent with those of Dufield et al. ${ }^{15}$ showing that the levels of aggrecan neoepitopes ARGS and AGEG were elevated in the synovial fluid of rat knee after intraarticular (IA) injection of TNF- $\alpha .^{31}$ Moreover, synovial fluid ARGS levels increased following surgically induced joint instability in the rat meniscal tear (MT) model. ${ }^{32}$ 
In a MIA-induced OA model in rats, serum glycosaminoglycan (GAG) levels significantly decreased after administration of diacetylrhein. ${ }^{33}$

Rats with arthritis induced by Freund's complete adjuvant (FCA) showed elevated levels of synovial fluid IL-1 $\beta$, IL-6, and TNF compared with the control group. In MIA rat model, a biphasic elevation of synovial fluid concentration of monocyte chemoattractant protein 1 (MCP-1), macrophage inflammatory protein $-3 \alpha$ (MIP-3 $\alpha$ ), and IL- 6 were observed. $^{34}$

\section{Mice}

In STR/ort mice, serum level of MMP-3 increased from week 7 to $9 .{ }^{35}$ In addition, high levels of urinary CTX-II were observed in the STR OA subgroup compared with the STR non-OA. ${ }^{36}$ In a study that compared wild-type (WT) and biglycan/fibromodulin double-deficient mice, the mean serum concentrations of $\mathrm{C} 2 \mathrm{C}$ and Coll2-1 were higher in biglycan/fibromodulin double-deficient mice than in the WT mice at all time points. Mean serum concentrations of Coll2-1NO increased progressively with age in WT mice but not in biglycan/fibromodulin double-deficient mice. ${ }^{37}$

In mice subjected to noninvasive ACL rupture via tibial compression, serum level of CTX-I was increased in injured mice at all time points compared to uninjured mice. In contrast, serum procollagen type $1 \mathrm{~N}$-terminal propeptide (P1NP) levels were not found to be significantly different between any of the experimental groups at any time points. ${ }^{8}$

COMP, MMP-3, and MMP-9 synovial fluid levels also increased in mice after acute joint injury induced by intraarticular fracture. ${ }^{38,39}$

In a study of Porta et al., ${ }^{40}$ the endocannabinoids anandamide (AEA) and 2-arachidonoylglycerol (2-AG) did not modify serum levels of MIA and control groups.

Other studies examined the involvement of inflammatory and oxidative stress biomarkers in the pathogenesis of OA. They demonstrated an increase in serum levels of IL-6, IL-1 $\beta$, and TNF- $\alpha$ both in STR/ort mice and in a model of intra-articular fracture. ${ }^{41,42}$ In obese and hyperlipidemic STR mice, serum levels of malondialdehyde (MDA), CTX-II and CPII were elevated compared with levels in the control CBA mice. ${ }^{43}$ Notably, the level of MDA was correlated with that of CTX-II, but not of CPII.

\section{Guinea Pigs}

The Dunkin-Hartley guinea pig strain displays a spontaneous onset of progressive degenerative changes in the knee joints that mimics the pathophysiological processes observed in primary human OA ${ }^{44,45} \mathrm{KS}$ and COMP levels were elevated in the knees of Hartley (OA-prone) compared with the strain 13 (OA-resistant) animals after 2 months of age. ${ }^{45,46}$ Moreover, synovial fluid concentration of KS and
COMP correlated positively with the severity of histological damage in both strains. ${ }^{46}$ In addition, the levels of serum $\mathrm{C} 2 \mathrm{C} / \mathrm{CPII}$, representing the ratio of type II collagen degradation and synthesis, were elevated in the OA-prone Hartley compared with strain 13 animal after 4 months of age. ${ }^{46,47}$ Huebner et al. ${ }^{47}$ observed abnormalities in collagen network beginning at 2 months of age. Among CTX-II, Coll21 , and $\mathrm{C} 2 \mathrm{C}$, only serum Coll2-1 increased coincidentally with the appearance of the early disruption of the collagen fibril and correlated with histological severity. ${ }^{47}$ In DunkinHartley guinea pig with ACLT, C2C, stromal cell-derived factor (SDF-1), GAG, MMP-13, and IL-1 $\beta$ were elevated in the synovial fluid compared with the control group. ${ }^{48}$ Significant positive correlations were found between the SDF-1, GAG, and C2C synovial fluid concentrations and the Mankin score. Huebner et al. ${ }^{46,49}$ showed that the Hartley strain exemplified a serum/chemokine profile indicative of a pro-inflammatory state compared with Strain 13. Among the cytokines and chemokines elevated in the Hartley strain, they found IL-6, IL-17, IL-8, and TNF- $\alpha$. In other research, they also demonstrated that transglutaminase isoenzyme 2 (TG2) concentrations were correlated with synovial fluid COMP. ${ }^{50}$

In another study, dietary omega-3 (n-3) polyunsaturated fatty acid (PUFA) reduced OA in the OA-prone DunkinHartley compared with OA-resistant Bristol Strain1-2 (BS2). ${ }^{51}$ McDougall et al. ${ }^{52}$ showed that urine concentrations of CTX-II were reduced by cathepsin $\mathrm{K}$ inhibitor. Horcajada et $a l .^{44}$ compared the effects of 3 polyphenols oleuropein, rutin, and curcumin on joint ageing and $\mathrm{OA}$ development. Coll2-1 was decreased by rutin and the combination of rutin/curcumin. Fib3-1 and Fib3-2 (2 specific peptides of fibulin-3) were only decreased by the rutin/curcumin mixture, while Coll2- $1 \mathrm{NO}_{2}$ was significantly decreased by all treatments. Moreover, Coll2-1 $\mathrm{NO}_{2}$, Fib31, and Fib3-2 were correlated with histological global OA score while Coll2-1 $1 \mathrm{NO}_{2}$ and Fib3-2 also correlated with synovial histological score. ${ }^{44}$ In a study of metabolic fingerprints, Lamers et al. ${ }^{53}$ showed that purine metabolism is of major importance in OA.

\section{Dogs}

In canines, synovial fluid CTX-II levels were elevated early after unilateral cruciate ligament transection. ${ }^{54}$ Coll2-1 and Coll2-1 $1 \mathrm{NO}_{2}$ also appeared to be OA biomarkers of early structural changes. Indeed, following ACL transection, a significant and rapid increase ( 2 weeks and 6 weeks, respectively) of these markers was observed in serum. ${ }^{55}$ Moreover, a positive and highly significant correlation was found between Coll2-1 concentrations and the global macroscopic score. Coll2-1 $1 \mathrm{NO}_{2}$ concentrations were found to be significantly and positively correlated with the size of the osteophytes. ${ }^{55}$ The ratio Coll2-1 $\mathrm{NO}_{2} / \mathrm{Coll2}-1$ was negatively correlated with 
macroscopic parameters and the histological score, but not with the osteophyte size.

In beagle dogs subjected to cranial cruciate ligament transection (CrCLt), synovial fluid levels of AGEG neoepitope were significantly elevated compared with either contralateral knee or contralateral knee in sham-operated animals. ${ }^{31}$ In a canine model of posttraumatic OA, serum level of KS and HA rise between 1 to 3 weeks after ACLT. ${ }^{56}$

In ACLT dog model, tenascin-C (TN-C), an extracellular matrix glycoprotein, increased in synovial fluid 1 month after ACLT compared with the control group. ${ }^{57}$

In dogs undergoing CrCLt, bone specific alkaline phosphatase (BAP) activity increased in synovial fluid with time after transection and zoledronic acid $(25 \mu \mathrm{g} / \mathrm{kg})$ partially inhibited this change. Synovial fluid C1,2C, CS, and serum CPII levels were significantly increased after transection. Zoledronic had no effects on these biomarkers. Interestingly, significant correlations were detected between synovial fluid BAP activity, serum CPII concentration and synovial fluid CS concentration and radiographic findings when all groups were combined. ${ }^{58}$ In a study published in 2009, Connor et al. ${ }^{59}$ showed that a cathepsin K inhibitor (SB-553484) reduced the levels of urinary CTX-I and CTX-II in mature female beagle dogs with partial medial meniscectomy. PF152, a novel highly selective MMP-13 inhibitor, reduced joint lesions in adult dogs with OA induced by partial MMx and decreased biomarkers of type II collagen neoepitope (TIINE) and aggrecan (neoepitopes ARGN or AGEG) degradation. ${ }^{60}$ In another study, high dose of vitamin E (400 UI/animal/day) administrated orally in $\mathrm{OA}$ dogs with $\mathrm{CrCL}$ transection, reduced inflammation markers including $\mathrm{NO}_{\mathrm{x}}, \mathrm{IL}-1 \beta$, and $\mathrm{PGE}_{2}$ in synovial fluid. ${ }^{61}$

During the progression of OA induced by a surgically created medial patellar luxation (MPL) in the left stifle of dog, synovial fluid and serum TIMP-2 levels were significantly decreased, whereas MMP-2 in synovial fluid was significantly increased. ${ }^{62}$ In dogs referred to the clinic, synovial fluid TIMP-1 level was increased in dogs with ACL rupture (ACLR), osteochondritis dissecans (OCD), fragmented coronoid process (FPC), and hip dysplasia (HD) compared to dogs without musculoskeletal diseases. ${ }^{63}$ Carprofen (2.2 and $4.4 \mathrm{mg} / \mathrm{kg}$ ), a nonsteroidal inflammatory drug, had no effect on MMP activity in ACLT-induced OA dogs. ${ }^{64}$ Synovial fluid PGE 2 levels were dose-dependently diminished by celecoxib in beagle dog OA induced in one knee according to the groove model showing a neutral effect of celecoxib on the characteristics of experimentally induced $\mathrm{OA}$ in vivo. ${ }^{65}$

In 2013, Gharbi et al. ${ }^{66}$ provided a proteome mapping of dog serum after ACLT surgery. A number of proteins, such as fetuin $\mathrm{B}$ and complement $\mathrm{C} 3$, were increased in $\operatorname{dog} \mathrm{OA}$ serum whereas others, such as hyaluronan binding protein 2, complement $\mathrm{C} 1 \mathrm{~s}$ and $\mathrm{C} 4$ were decreased. ${ }^{66}$
Another study investigated the effects of total MMx on biomarkers in synovial lavage fluid. COMP and KS were significantly increased. ${ }^{67}$ Serum levels of COMP and urinary levels of CTX-II were also increased after a joint bleed mimicked by injecting freshly collected (vena puncture) autologous blood intra-articularly in the knee. ${ }^{68}$ Another research observed that a subcutaneous injection of calcitonin (3 units per kg of body weight) significantly decreased the urinary excretion of D-pyridinoline (D-Pyr) and blood levels of KS and HA in dogs after ACLT. ${ }^{69}$

\section{Horses}

The published studies investigated the effect of exercises on OA incidence and progression. A study showed that the subchondral bone lysis on standard X-ray was strongly correlated with CPII, and that subchondral bone proliferation at the joint capsule attachment was strongly correlated with $\mathrm{PGE}_{2}{ }^{70}{ }^{70}$ In addition, radial carpal bone edema detected using MRI correlated well with $\mathrm{C} 1,2 \mathrm{C}$ increase in the synovial fluid. Synovial fluid levels of Coll2-1 were significantly elevated in tarsocrural joints affected with osteochondrosis compared with unaffected joints. ${ }^{71,72}$ Another study compared sera of box-stall rest, pasture and trained foals. ${ }^{73}$ Lower serum levels of CPII and higher levels of CTX-I were found in the trained foals compared with the other groups. It was noticed that serum levels of CTX-I and CPII significantly increased with age in all groups. ${ }^{73}$ In another study, exercise induced a significant increase in the synovial fluid CPII, GAG, C1C, C2C, CTX1 concentrations. ${ }^{74}$ Finally, Fuller et al. ${ }^{75}$ in 2010 demonstrated that levels of synovial fluid BAP were significantly higher in the clinically active joint than in the contralateral joint, while $\mathrm{KS}, \mathrm{GAG}$, and $\mathrm{HA}$ were significantly lower in OA than in the contralateral joint. These biomarkers also showed good correlation with arthroscopy score of cartilage damage.

In an equine metacarpophalangeal groove model, Manninchedda et al. ${ }^{76}$ showed that synovial fluid levels of C2C, COMP, CTX-I, osteocalcin (OC), and CS-846 were not significantly different between groups. ${ }^{76}$

\section{Sheep}

In sheep undergoing meniscal destabilization (MD), increased levels of dimethyl sulfone $\left(\mathrm{DMSO}_{2}\right)$ were observed in serum after 4 weeks by nuclear magnetic resonance spectroscopy, while in ACLT-induced OA sheep, an increase of 3-methylhistidine and a decrease of branched chain amino acids, comprising the essential amino acids valine, leucine, and isoleucine (BCAAs) were identified. ${ }^{77}$ In another study, synovial fluid of MMP-2 was detected 2 and 4 weeks postoperatively in a temporomandibular joint (TMJ) model of OA in sheep. ${ }^{78}$ Moreover, MMP-2 activity correlated with initial articular cartilage destruction rather 
than with the progression of OA. In sheep responses to ACL reconstruction injury, a metabolic profiling of synovial fluid identified 6 metabolites such as isobutyrate, glucose, hydroxyproline, asparagine, serine, and uridine, which could be suggested as biomarkers for early postinjury degenerative changes in the knee joints. ${ }^{79}$

\section{Discussion}

We have attempted to gather all animal studies in English and French published between 1995 and 2015 and investigating soluble biomarkers in OA. A summary is available in Table 1. We also proposed a classification scheme for these biomarkers, largely inspired of the BIPEDS classification (Table 1).

In all animal studies that we have selected, soluble biomarkers are viewed as useful indicators of joint tissue metabolism, particularly of bone and cartilage. Some of them are well correlated with histological lesion severity, and can be considered as a burden of disease biomarkers, but none of them can be classified in the prognostic and safety categories. ${ }^{10}$

One major concern in animal studies is the method to harvest synovial fluid. Although the collection of synovial fluid in animal models can be challenging, synovial fluid offers the most proximal source for joint turnover information. ${ }^{80}$ The majority of studies in animals have utilized the technique of joint lavage. However, other methods exist for collecting specimens depending on the animal size. In small animal like mice, rats or guinea pigs, Whatman paper can be used to absorb synovial fluid. ${ }^{39}$ Recently, Seifer et al. ${ }^{39}$ described a method for synovial fluid recovery using Melgisorb (Tendra, Sweden), a calcium sodium alginate compound (CSAC), used as a dressing on wounds to absorb heavy exudate. In dogs, joint lavage with saline has been used to collect synovial fluid and the dilution effect of the lavage has been corrected by normalization to serum urea. ${ }^{80}$

Another concern is the age of animals used and the presence or not of cartilage growth plate. In fact, some of the studies reported in this review were conducted in young immature animals, meaning that biomarker levels can be confounded by the presence of growth plate. This should be keep in mind when interpreting results.

\section{Burden of Disease Biomarkers}

Numerous biomarkers measured in serum or synovial fluids were correlated with histologic or macroscopic cartilage lesions severity score in different species like rat, mouse, $\mathrm{dog}$, or guinea pig. Interestingly, some of them increased rapidly after the onset of the disease, before the appearance of the histological structural changes. Most particularly, Coll2-1 is particularly precocious. In serum of guinea pig, Coll2-1 increase was coincident with the early disruption of the collagen fibril visible by birefringence before the appearance of structural changes observable by conventional microscopy. This finding suggests that the Coll2-1 biomarker may be an early indicator of the onset of chondropathy in the guinea pig. ${ }^{47}$ This also means that biomarkers of type II collagen degradation could be considered as surrogate biomarkers of histology in animal study. This could be advantageous for studying the kinetic of drug candidate efficacy and reduce the number of euthanized animals.

\section{Prognostic Biomarkers}

Demonstrating the prognostic value of biomarkers in animal models remains very challenging because in most models, OA is induced using well-standardized methods and lesion progression is predictable and homogenous. To be recognized as a prognostic biomarker, a biomarker has to predict the incidence or the progression of the disease. To our knowledge, there is no biomarker that can be classified in this category based on animal study. This would require a prospective study including pets with OA risk factors and different disease progression rate. This type of information could be helpful for the daily management of care in pets.

\section{Efficacy of Intervention}

Soluble biomarkers have been largely used as drug development tools in OA. Not exhaustively, we can mention in rats, diacetylrhein that reduces serum GAG levels, ${ }^{33}$ oral glucosamine hydrochloride that prevents the increase of CTX-II levels ${ }^{23}$ and alendronate, a potent inhibitor of osteoclastic bone resorption that decreases serum levels of COMP. ${ }^{24}$ In guinea pigs, prolonged (1 month) administration of cathep$\sin \mathrm{K}$ inhibitor reduces UCTX-II excretion and oleuropein decreases Coll2-1. ${ }^{44,52}$ In dogs, cathepsin $\mathrm{K}$ decreases urinary CTX-I and CTX-II ${ }^{59}$ and PF152 reduces TIINE and aggrecan (neoepitopes ARGN or AGEG) ${ }^{60}$ Another study in dogs with OA using a supplement with a high dose of vitamin $\mathrm{E}$ showed a reduction in inflammation joint markers such as PGE ${ }_{2}$ and IL-1 $\beta .{ }^{61}$ These findings indicate that a large panel of biomarkers should be used in the preclinical phase of drugs or other intervention development. This approach would allow a better understanding of their mechanisms of action but also the early detection of potential safety issues, and hence could enhance the drug development efficiency, and possibly reduce the overall cost of OA clinical trial. This also allows the selection of one or more biomarkers sensitive to drug activity that can be used in the next phases of drugs development. For these reasons, the US Food and Drug Administration (FDA), the Osteoarthritis Research Society International (OARSI) and European Medicine Agency (EMA), recently published guidelines recommending a higher level of integration of biomarkers 


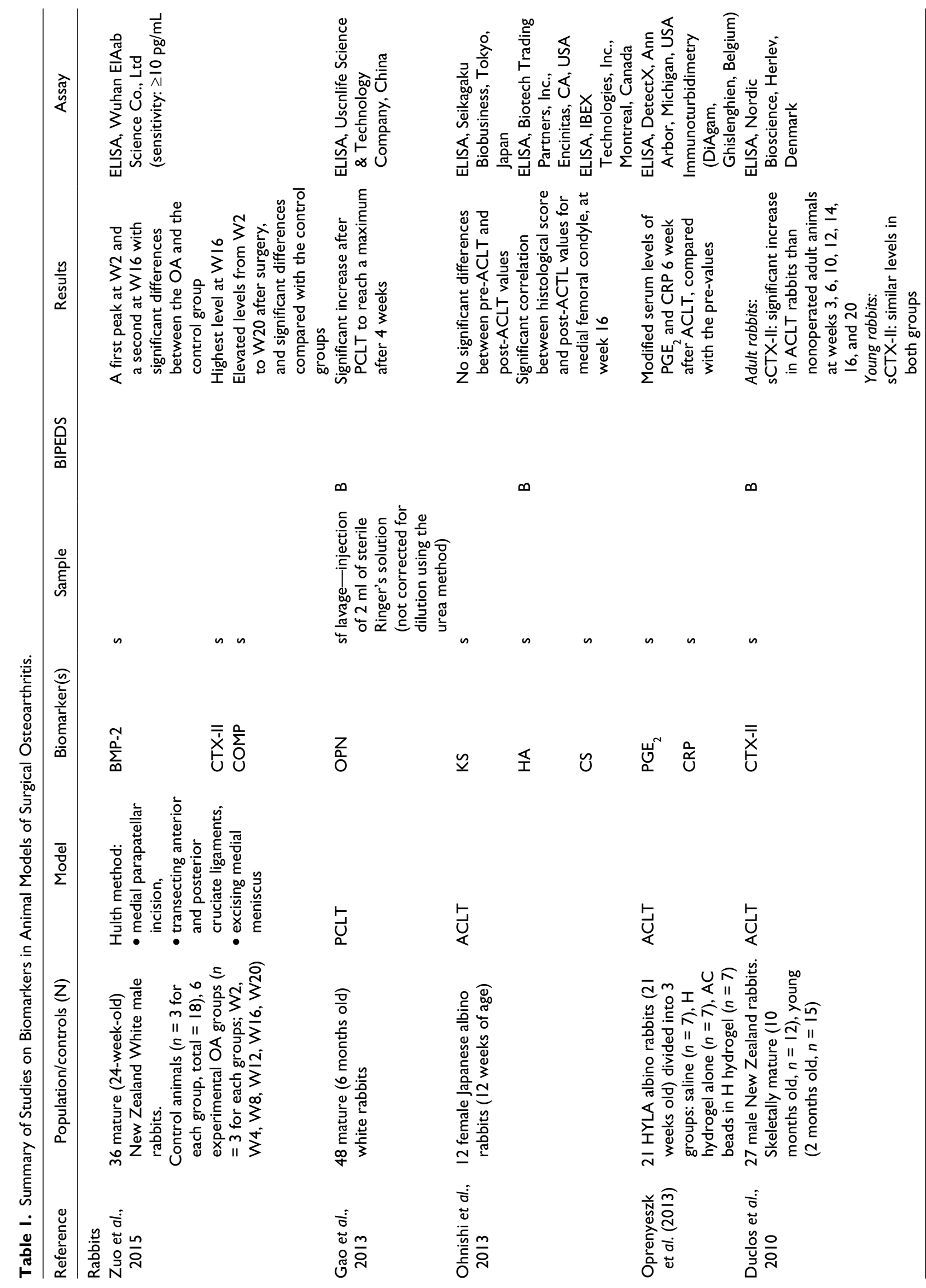




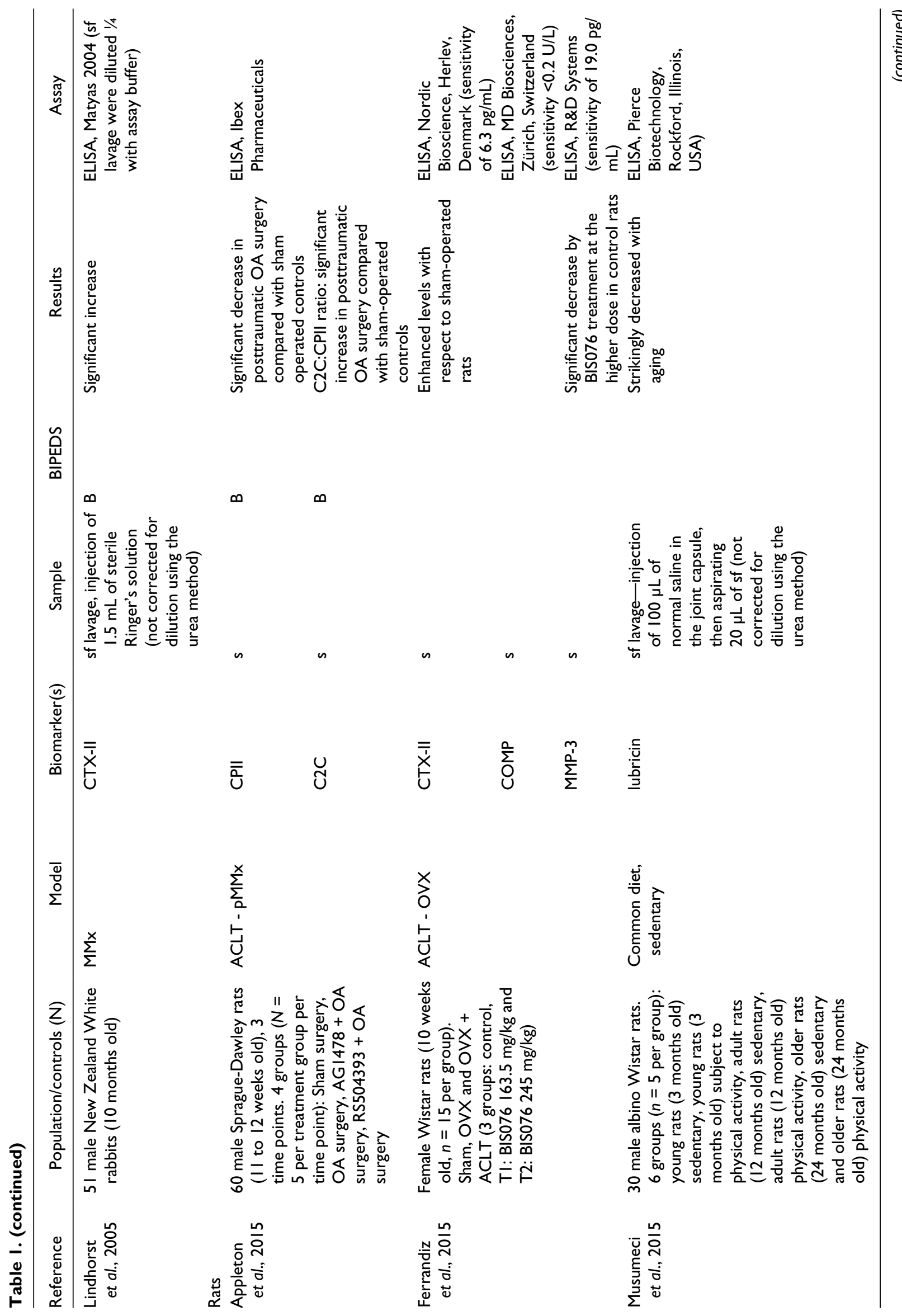




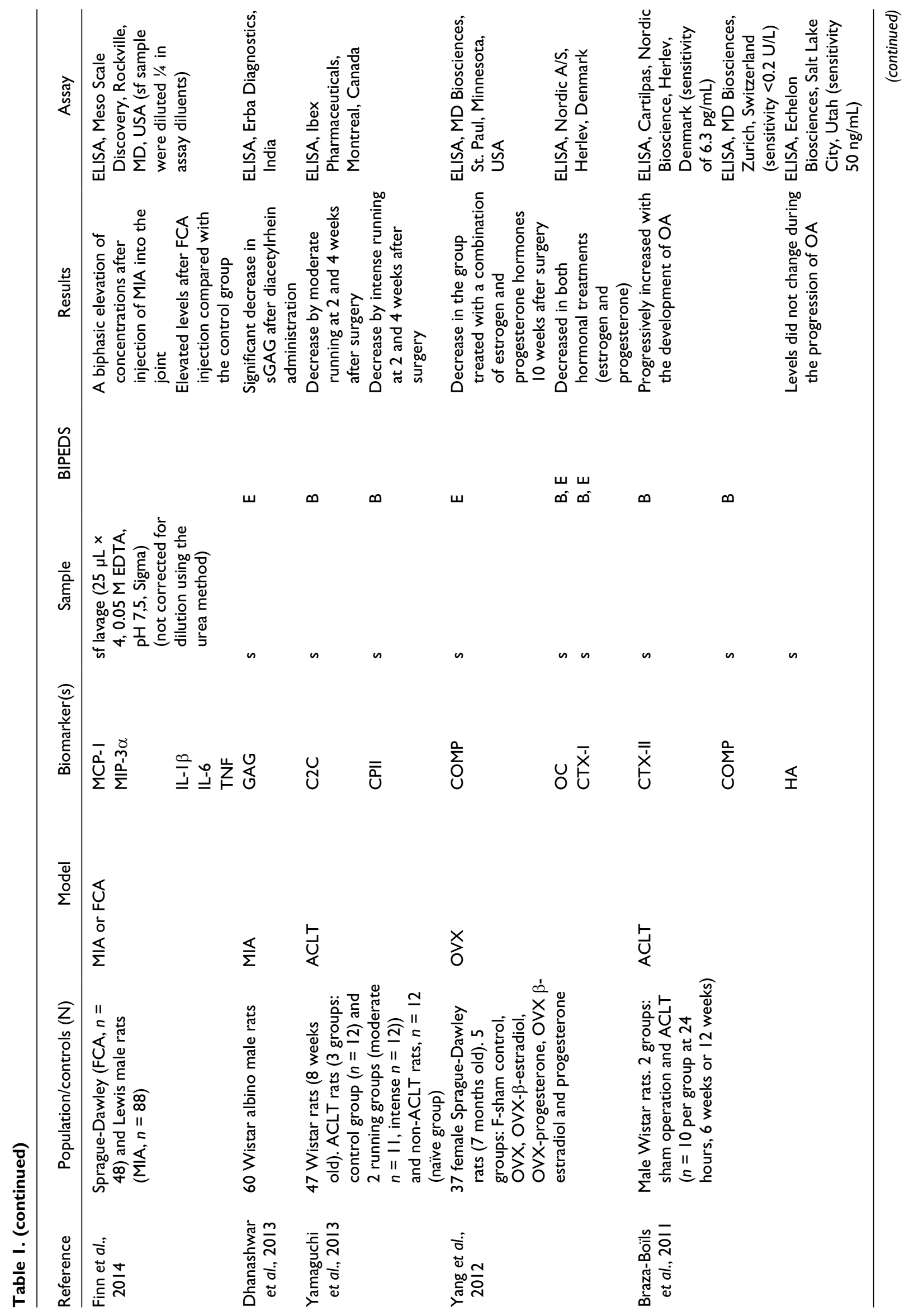




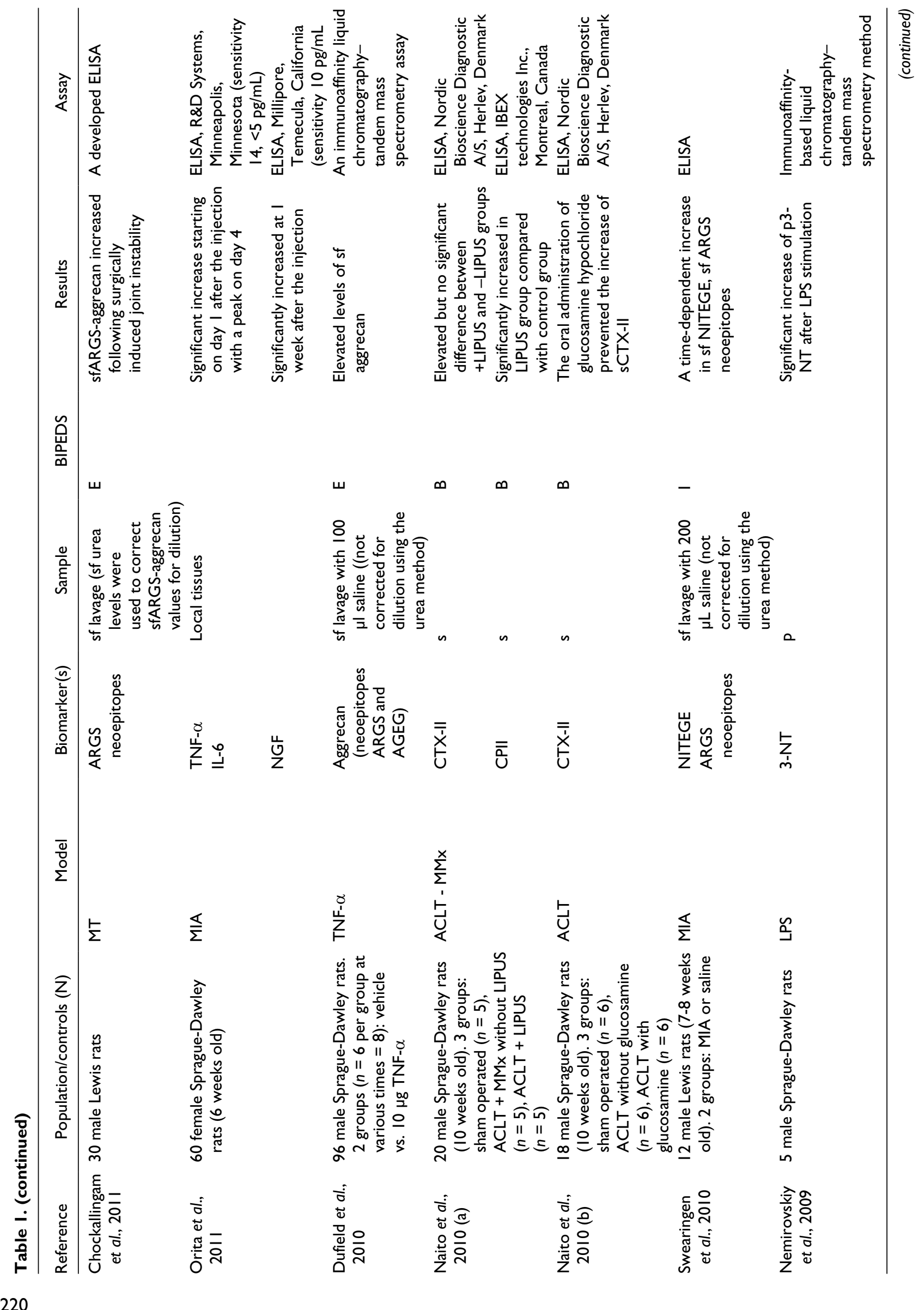




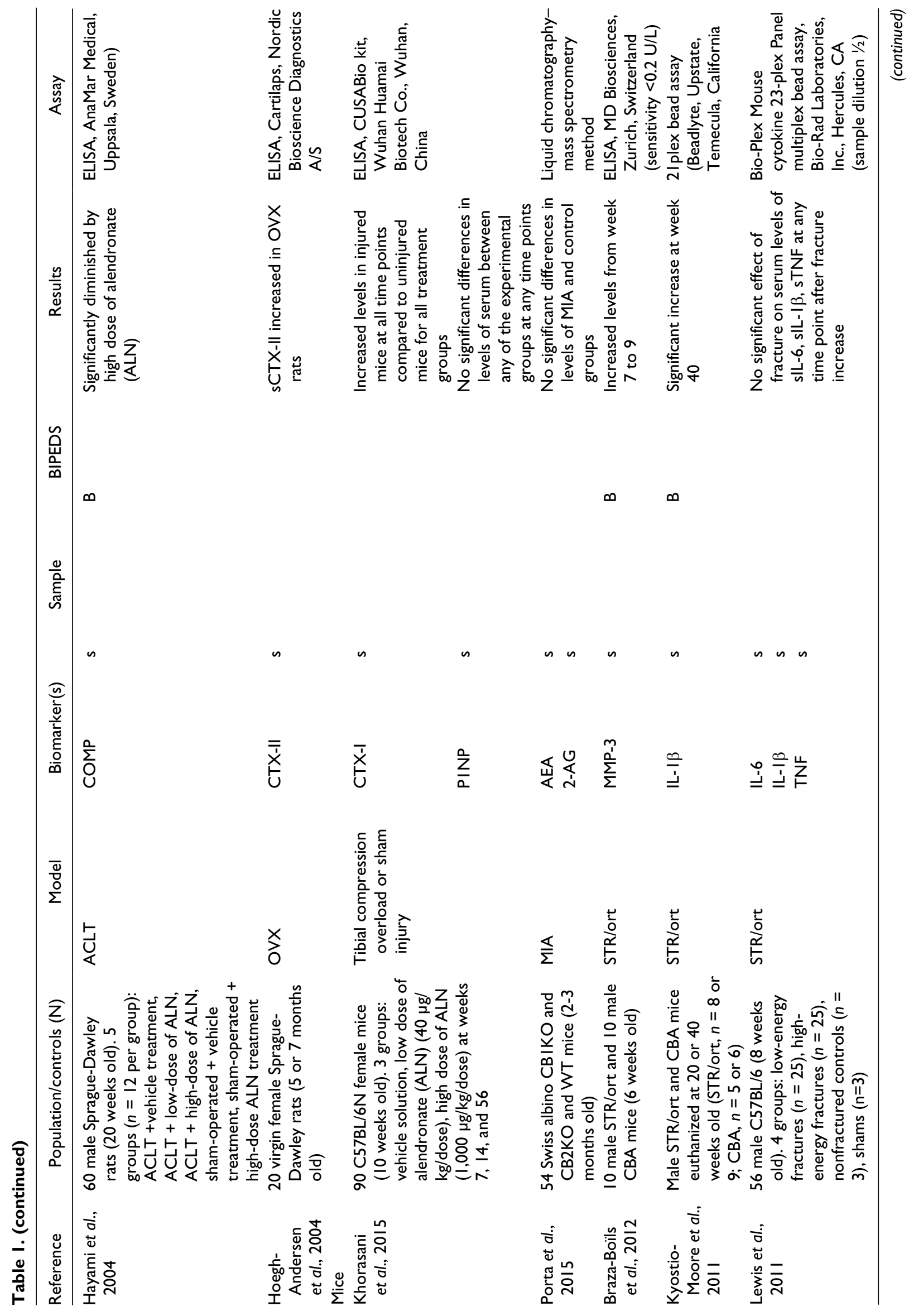




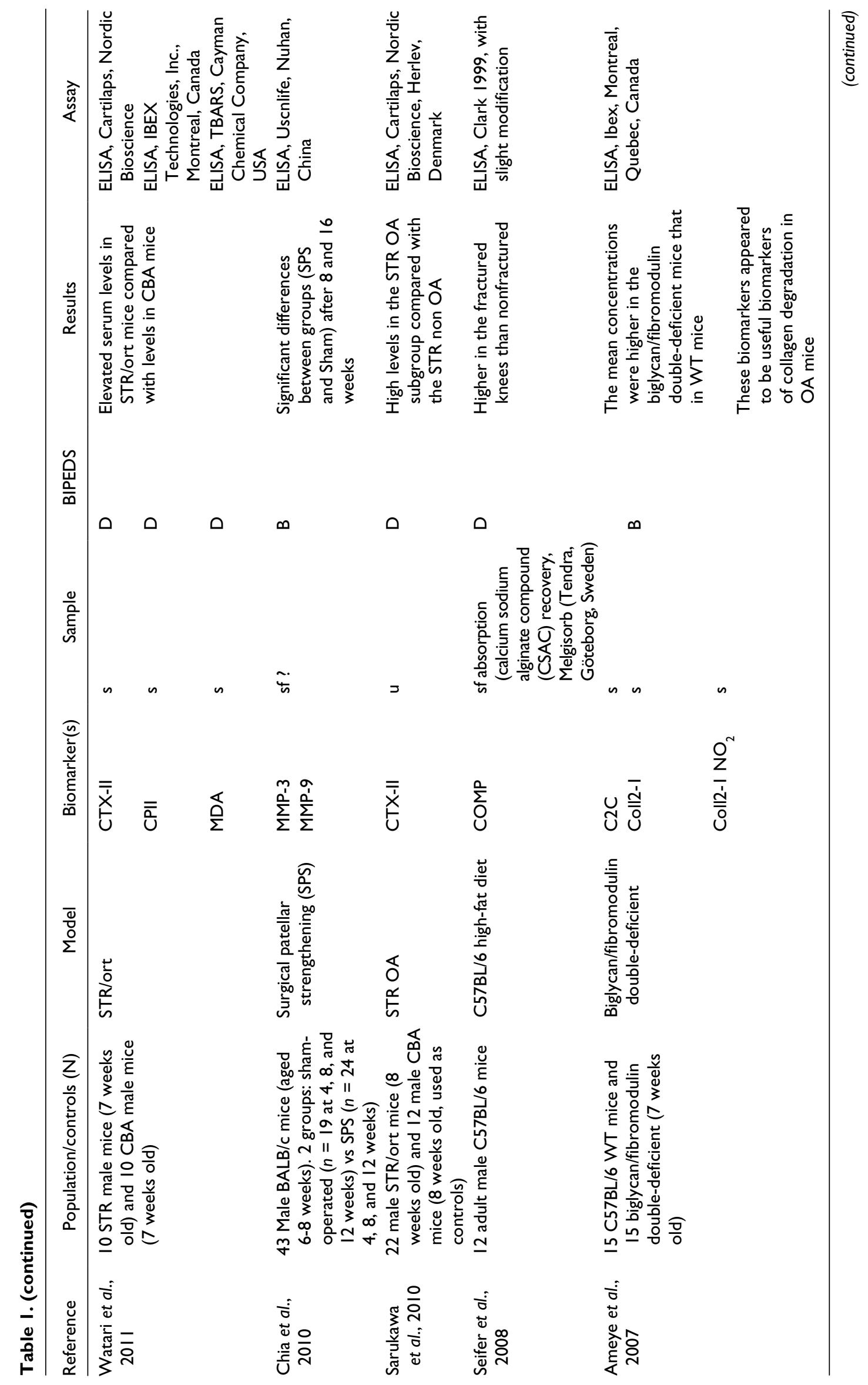




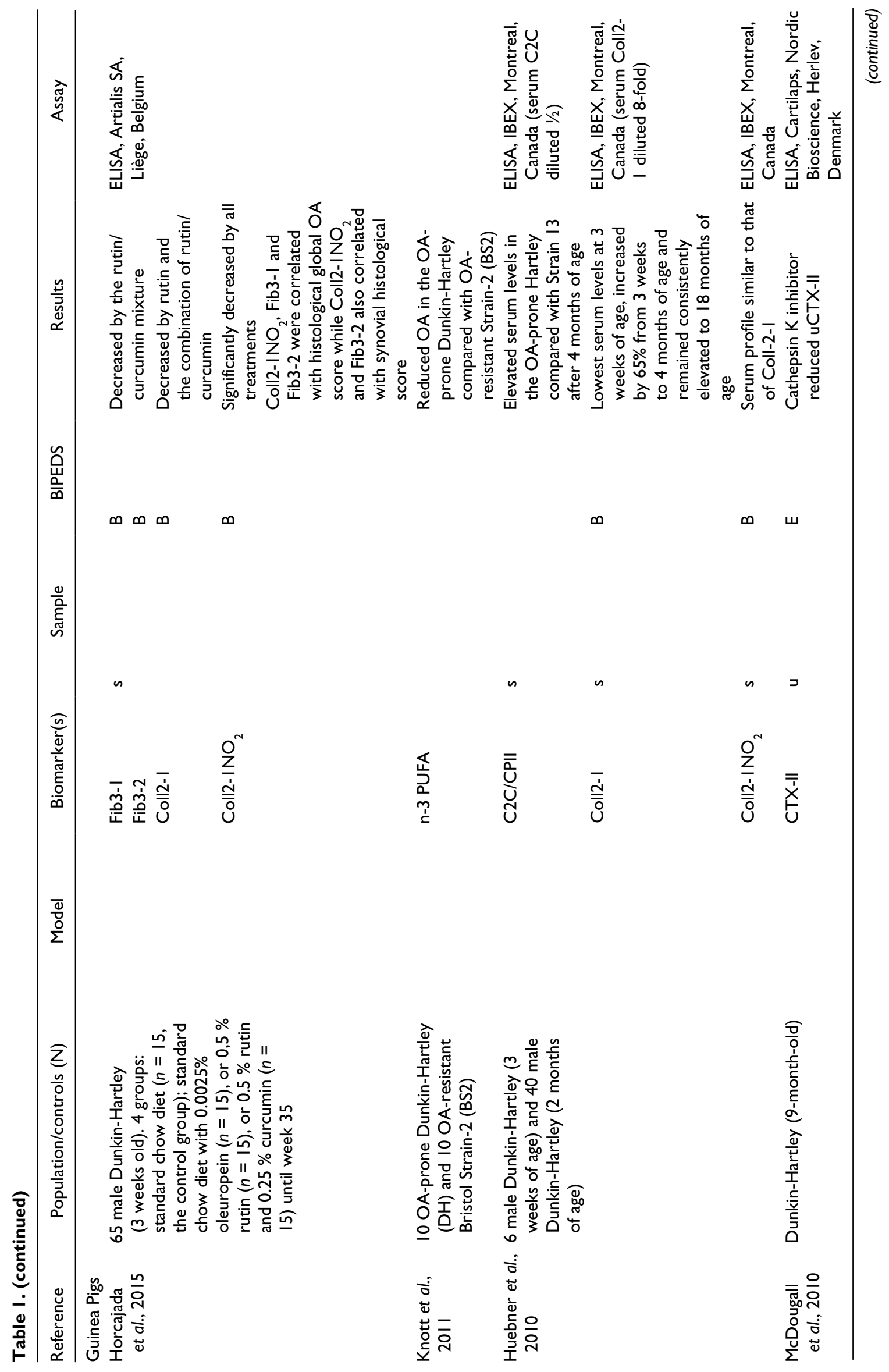




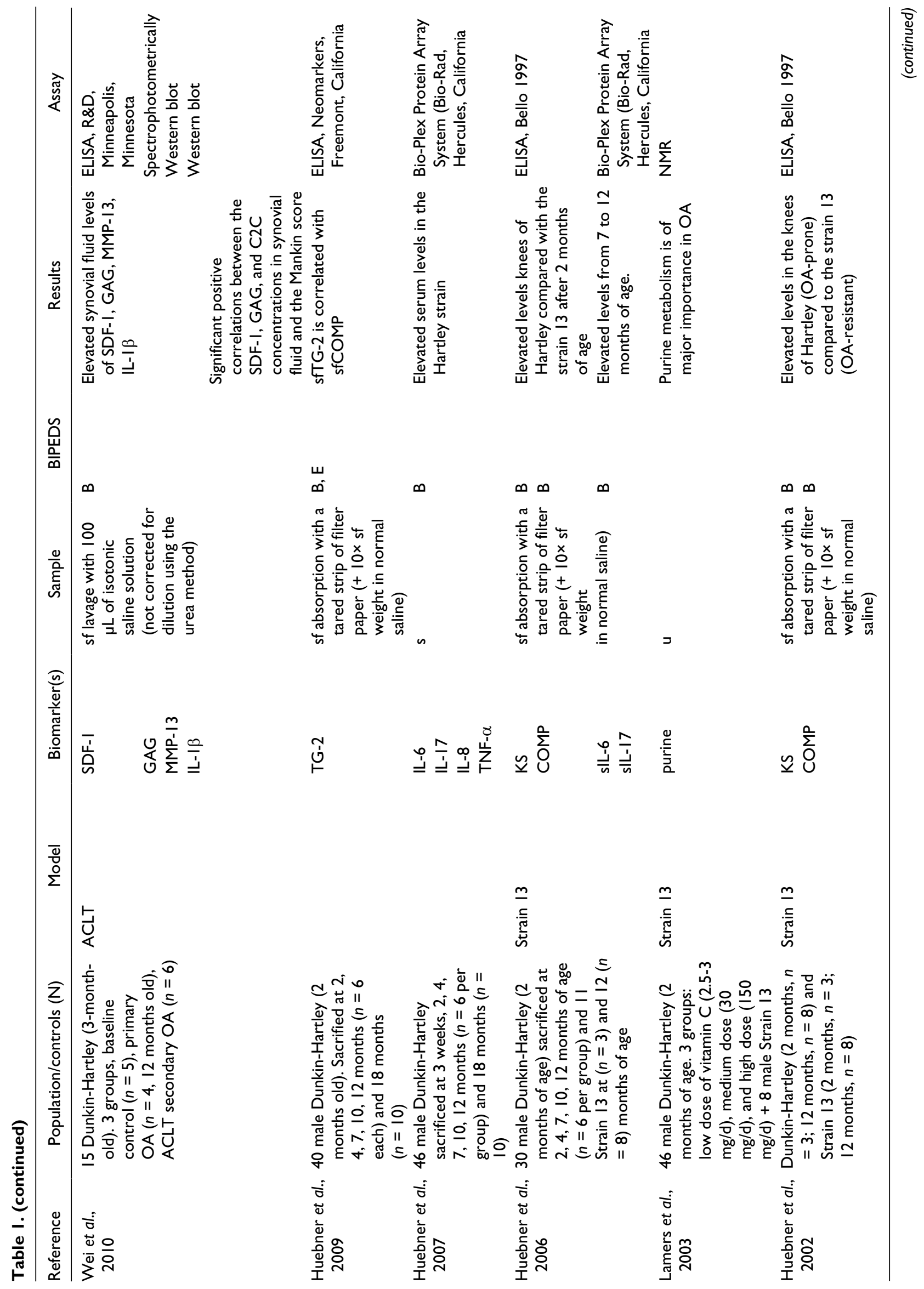




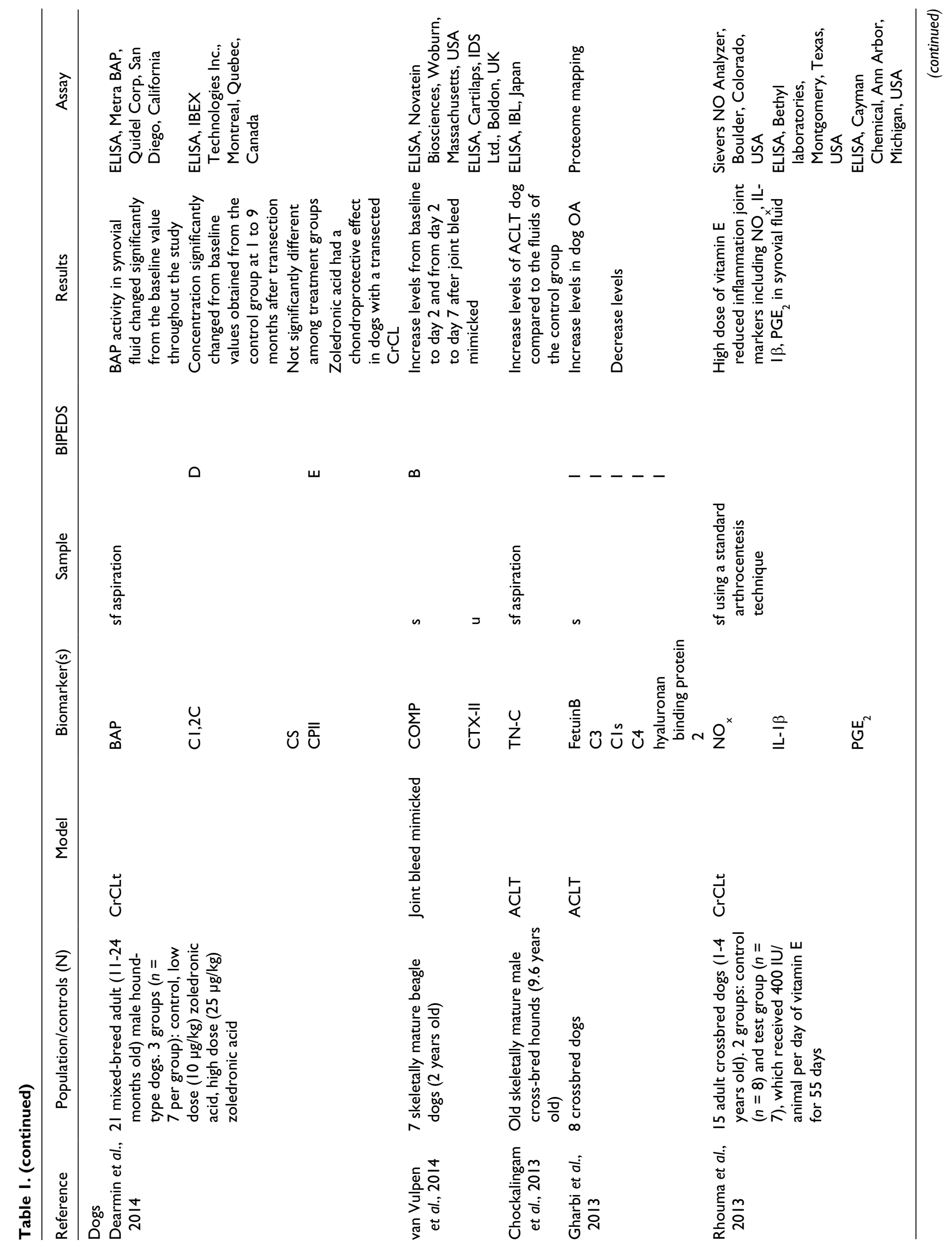




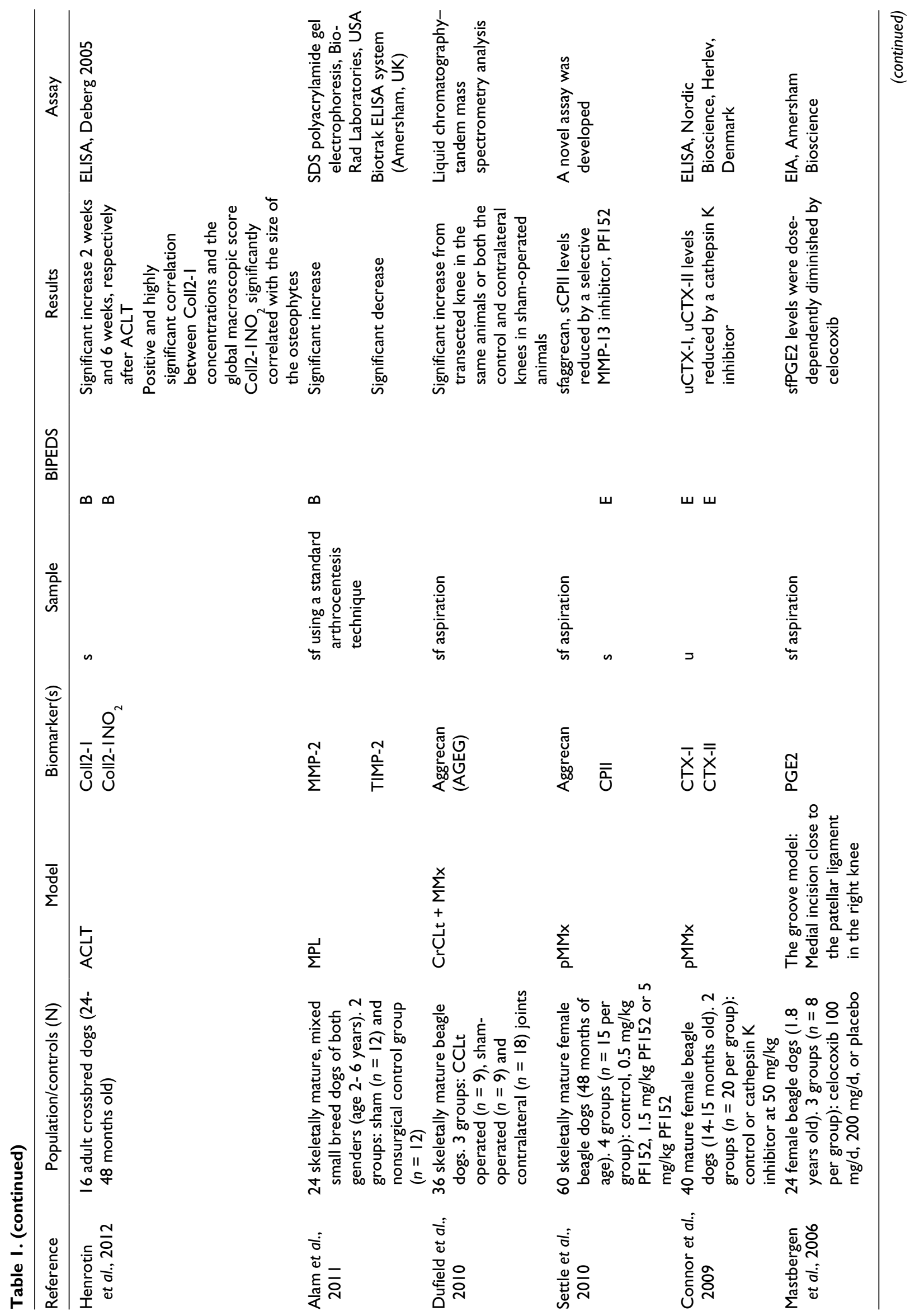




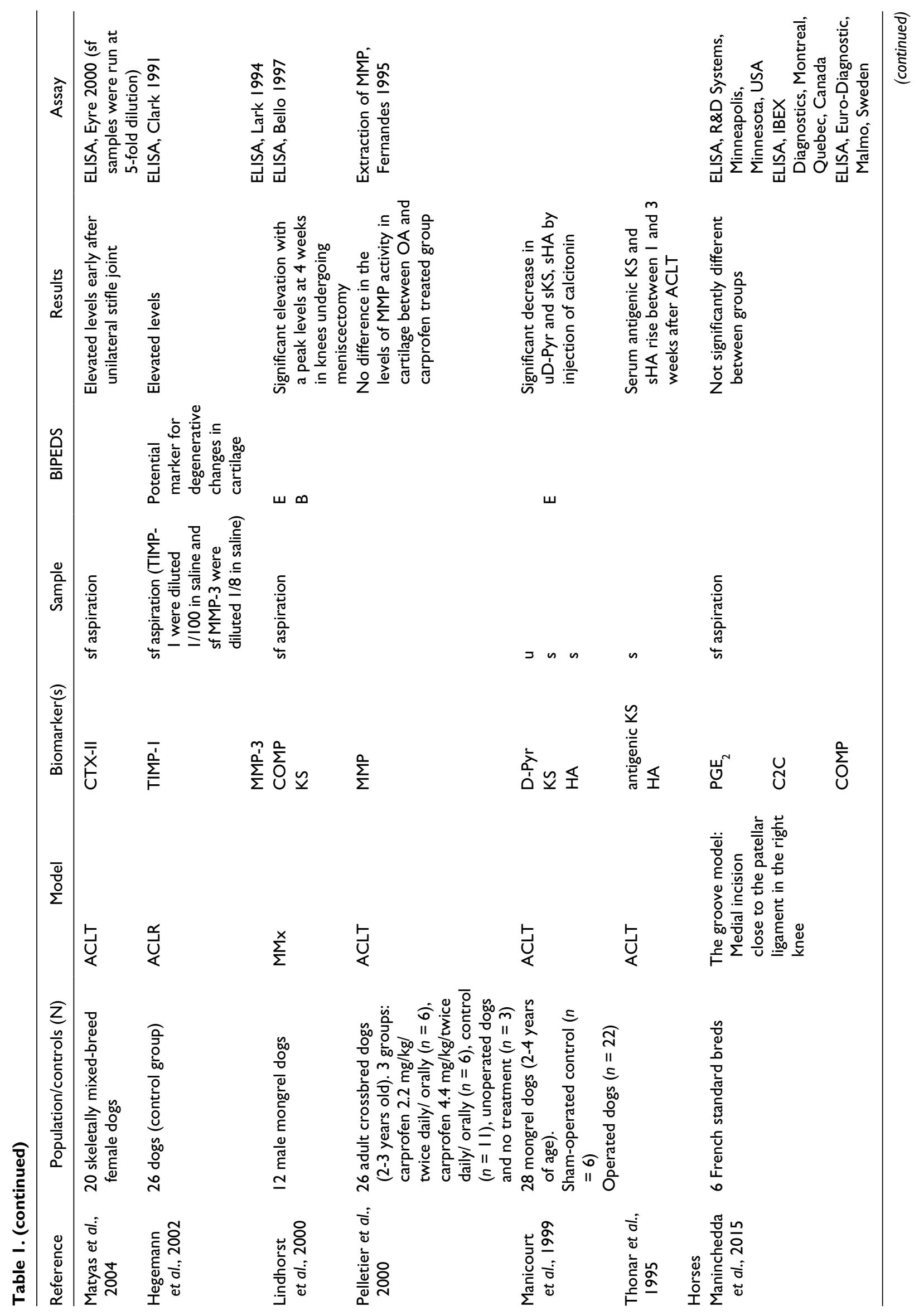




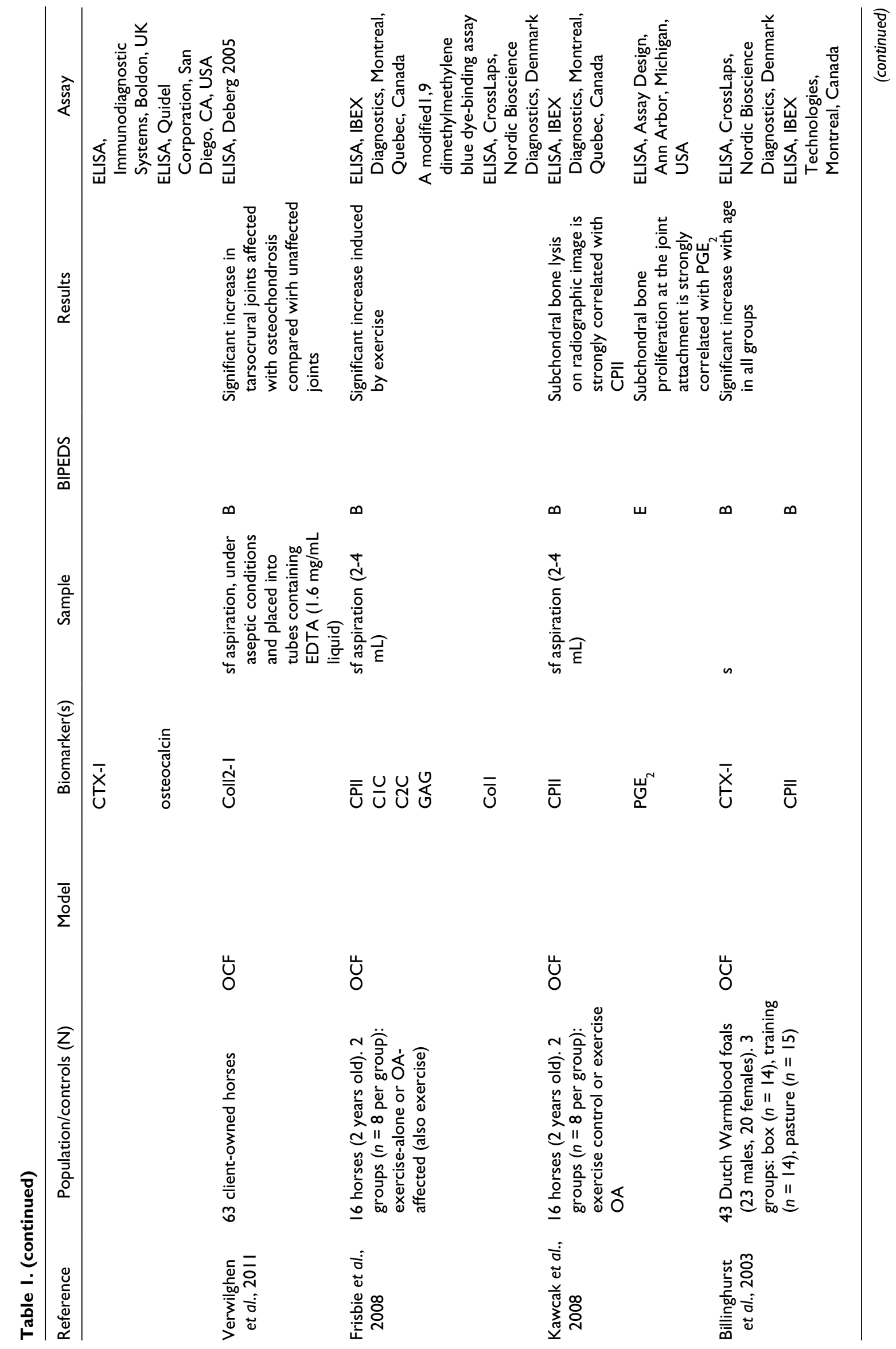




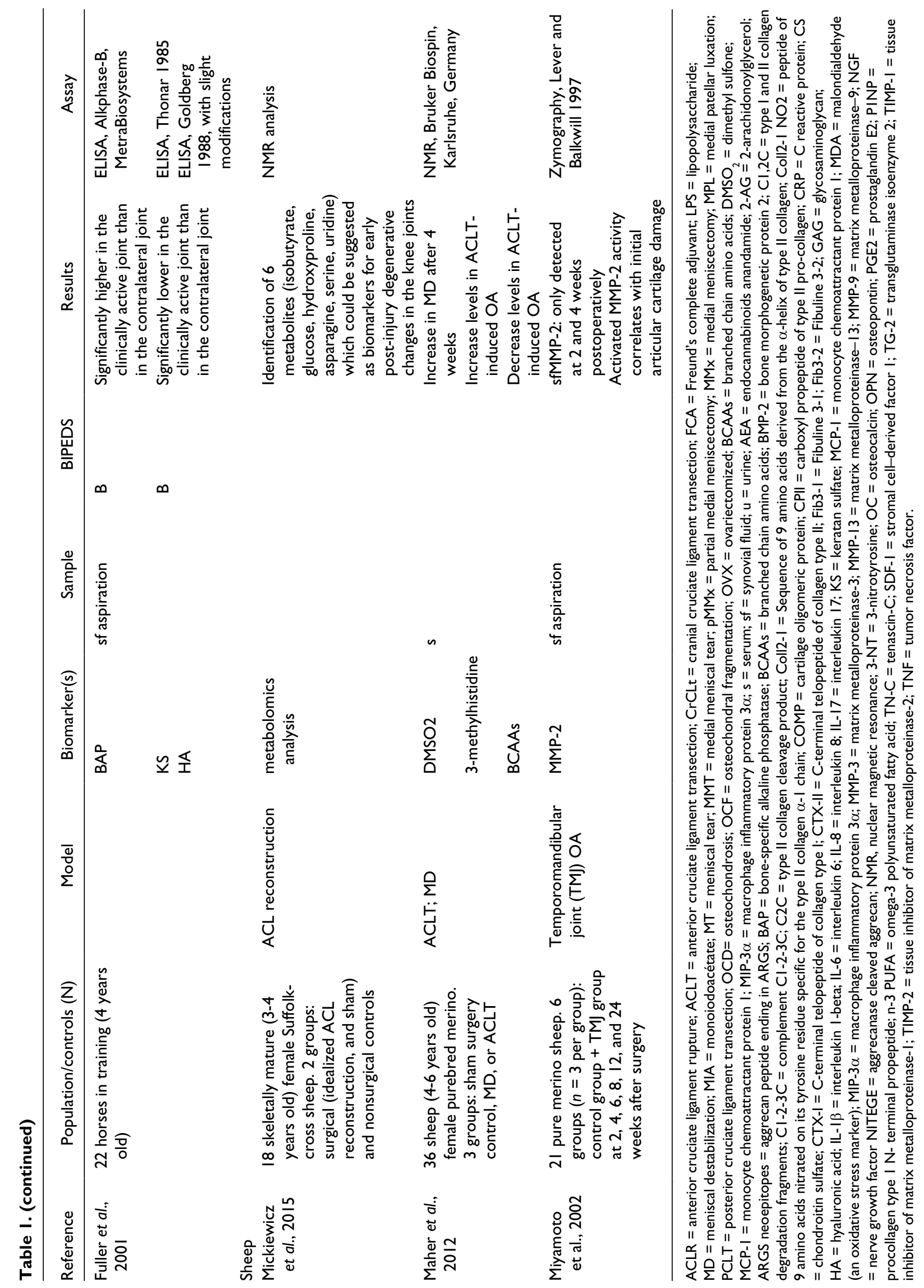


in the development and testing of new drugs to advance decision making on dosing, time and treatment effect, trial design, and risk/benefit analysis. ${ }^{3}$

\section{Diagnostic Biomarkers}

Diagnostic biomarkers ideally differentiate animals with and without the disease. At this time, in guinea pigs, only the ratio $\mathrm{C} 2 \mathrm{C} / \mathrm{CPII}$, reflecting type II collagen turnover, have been demonstrated to discriminate OA-prone Hartley and strain 13 animals, a strain that does not develop OA. ${ }^{46}$ This ratio was higher in OA-prone Hartley strain guinea pigs compared to OA-resistant strain 13 , indicating an upregulation of collagen degradation in the absence of a compensatory upregulation in collagen synthesis between 2 and 12 months of age. ${ }^{46}$ In mice, several biomarkers discriminate different mouse strain. Serum levels of MDA, CTX-II and CPII were elevated in obese and hyperlipidemic STR/ORT (STR) mice compared with control CBA mice. ${ }^{43}$ Together, these observations suggest that oxidative stress is associated with the development of OA, possibly via the degradation of type II collagen.$^{43}$ In parallel, high levels of urinary CTX-II were found in the STR OA subgroup compared with the STR non-OA. ${ }^{36}$ The models are very relevant to validate biomarker and assess their capacity to discriminate diseased and nondiseased animals.

\section{Safety Biomarkers}

In 2011, the BIPED classification was expanded and a "Safety" category to the BIPED system was added. ${ }^{81}$ Safety biomarkers could be used in preclinical and clinical applications to monitor the health of the joint tissues, the whole joint organ, or the skeleton in general. ${ }^{81}$ For instance, biomarkers reflecting the synthesis of the main proteins of the joint might provide an index of the "joint-protective" effect of a potential treatment. ${ }^{81}$ Currently, there are no studies exploring specifically this aspect of joint tissue related biomarkers. A case in point could be evaluation of fibrosis markers in animals treated with MMP inhibitors.

\section{Conclusion}

In conclusion, this systematic review indicates that some markers could be valuable to evaluate the burden of OA disease and to assess the therapeutic response in animal models. The most investigated biomarkers in terms of burden of disease assessment are those derived from type II collagen metabolism. Since OA represents a process of matrix damage, turnover, and attempted repair, the use of multiple biomarkers, both anabolic and catabolic, could likely be most accurate in characterizing OA. ${ }^{11}$ Finally, soluble biomarkers can be a useful "drug development tools" that should be early integrated in the research program of new drugs. As previously mentioned, biomarkers may be helpful to assess drug safety, to assist with selection of animal models and lead compounds and assess drug mechanisms of action. ${ }^{3}$ Furthermore, the use of biomarkers at the preclinical stages is helpful to select the doses and the route of administration, to evaluate the residency time in joint, to select a companion biomarker for the next clinical phases and the personalized management of $\mathrm{OA}$, to provide insights into disease pathophysiology and to provide confidence in histological assessment (when synovial fluid markers know to correlate with histology provide consistent results with histology in a subsequent study).

\section{Acknowledgments and Funding}

The author(s) received no financial support for the research, authorship, and/or publication of this article.

\section{Declaration of Conflicting Interests}

The author(s) declared the following potential conflicts of interest with respect to the research, authorship, and/or publication of this article: Y. E. Henrotin is the founder of Artialis SA, a spin-off of the University of Liège. C. Sanchez and Y. E. Henrotin have received funding from the European Union. The D-Board project has received funding from the European Union's Seventh Framework Programme for research, technological development, and demonstration under grant agreement no. 305815.

\section{References}

1. Bauer DC, Hunter DJ, Abramson SB, Attur M, Corr M, Felson D, et al. Classification of osteoarthritis biomarkers: a proposed approach. Osteoarthritis Cartilage. 2006;14(8):7237. doi:10.1016/j.joca.2006.04.001.

2. Lotz M, Martel-Pelletier J, Christiansen C, Brandi ML, Bruyere O, Chapurlat R, et al. Republished: Value of biomarkers in osteoarthritis: current status and perspectives. Postgrad Med J. 2014;90(1061):171-8. doi:10.1136/postgradmedj-2013-203726rep.

3. Kraus VB, Blanco FJ, Englund M, Henrotin Y, Lohmander LS, Losina E, et al. OARSI Clinical Trials Recommendations: soluble biomarker assessments in clinical trials in osteoarthritis. Osteoarthritis Cartilage. 2015;23(5):686-97. doi:10.1016/j.joca.2015.03.002.

4. Henrotin Y, Addison S, Kraus V, Deberg M. Type II collagen markers in osteoarthritis: what do they indicate? Curr Opin Rheumatol. 2007;19(5):444-50. doi:10.1097/ BOR.0b013e32829fb3b5.

5. Kraus VB. Osteoarthritis year 2010 in review: biochemical markers. Osteoarthritis Cartilage. 2011;19(4):346-53. doi:10.1016/j.joca.2011.02.002.

6. van Spil WE, DeGroot J, Lems WF, Oostveen JC, Lafeber FP. Serum and urinary biochemical markers for knee and hiposteoarthritis: a systematic review applying the consensus BIPED criteria. Osteoarthritis Cartilage. 2010;18(5):605-12. doi:10.1016/j.joca.2010.01.012.

7. Ward BD, Furman BD, Huebner JL, Kraus VB, Guilak $\mathrm{F}$, Olson SA. Absence of posttraumatic arthritis following 
intraarticular fracture in the MRL/MpJ mouse. Arthritis Rheum. 2008;58(3):744-53. doi:10.1002/art.23288.

8. Khorasani MS, Diko S, Hsia AW, Anderson MJ, Genetos DC, Haudenschild DR, et al. Effect of alendronate on posttraumatic osteoarthritis induced by anterior cruciate ligament rupture in mice. Arthritis Res Ther. 2015;17(1):30. doi:10.1186/s13075-015-0546-0.

9. Malemud CJ. Markers of osteoarthritis and cartilage research in animal models. Curr Opin Rheumatol. 1993;5(4):494-502.

10. Poole R, Blake S, Buschmann M, Goldring S, Laverty $\mathrm{S}$, Lockwood S, et al. Recommendations for the use of preclinical models in the study and treatment of osteoarthritis. Osteoarthritis Cartilage. 2010;18(Suppl 3):S10-6. doi:10.1016/j.joca.2010.05.027.

11. Teeple E, Jay GD, Elsaid KA, Fleming BC. Animal models of osteoarthritis: challenges of model selection and analysis. AAPS J. 2013;15(2):438-46. doi:10.1208/s12248-0139454-x.

12. Oprenyeszk F, Chausson M, Maquet V, Dubuc JE, Henrotin Y. Protective effect of a new biomaterial against the development of experimental osteoarthritis lesions in rabbit: a pilot study evaluating the intra-articular injection of alginate-chitosan beads dispersed in an hydrogel. Osteoarthritis Cartilage. 2013;21(8):1099-107. doi:10.1016/j.joca.2013.04.017.

13. Ohnishi A, Osaki $T$, Matahira $Y$, Tsuka $T$, Imagawa $T$, Okamoto $\mathrm{Y}$, et al. Evaluation of the chondroprotective effects of glucosamine and fish collagen peptide on a rabbit ACLT model using serum biomarkers. J Vet Med Sci. 2013;75(4):421-9. doi:10.1292/jvms.12-0240.

14. Gao SG, Cheng L, Zeng C, Wei LC, Zhang FJ, Tian J, et al. Usefulness of specific OA biomarkers, thrombin-cleaved osteopontin, in the posterior cruciate ligament OA rabbit model. Osteoarthritis Cartilage. 2013;21(1):144-50. doi:10.1016/j.joca.2012.09.006.

15. Duclos ME, Roualdes O, Cararo R, Rousseau JC, Roger T, Hartmann DJ. Significance of the serum CTX-II level in an osteoarthritis animal model: a 5-month longitudinal study. Osteoarthritis Cartilage. 2010;18(11):1467-76. doi:10.1016/j. joca.2010.07.007.

16. Zuo H, Jiang L, Qu N, Wang J, Cui X, Yao W. The biomarkers changes in serum and the correlation with quantitative MRI markers by histopathologic evaluation of the cartilage in surgically-induced osteoarthritis rabbit model. PLoS One. 2015;10(4):e0124717. doi:10.1371/journal.pone.0124717.

17. Lindhorst E, Wachsmuth L, Kimmig N, Raiss R, Aigner T, Atley L, et al. Increase in degraded collagen type II in synovial fluid early in the rabbit meniscectomy model of osteoarthritis. Osteoarthritis Cartilage. 2005;13(2):139-45. doi:10.1016/j. joca.2004.10.017.

18. Gerwin N, Bendele AM, Glasson S, Carlson CS. The OARSI histopathology initiative-recommendations for histological assessments of osteoarthritis in the rat. Osteoarthritis Cartilage. 2010;18(Suppl 3):S24-34. doi:10.1016/j.oca.2010.05.030.

19. Hoegh-Andersen P, Tanko LB, Andersen TL, Lundberg CV, Mo JA, Heegaard AM, et al. Ovariectomized rats as a model of postmenopausal osteoarthritis: validation and application. Arthritis Res Ther. 2004;6(2):R169-80. doi:10.1186/ar1152.
20. Yamaguchi S, Aoyama T, Ito A, Nagai M, Iijima H, Zhang $\mathrm{X}$, et al. Effects of exercise level on biomarkers in a rat knee model of osteoarthritis. J Orthop Res. 2013;31(7):1026-31. doi:10.1002/jor.22332.

21. Braza-Boils A, Alcaraz MJ, Ferrandiz ML. Regulation of the inflammatory response by tin protoporphyrin IX in the rat anterior cruciate ligament transection model of osteoarthritis J Orthop Res. 2011;29(9):1375-82. doi:10.1002/jor.21411.

22. Naito K, Watari T, Muta T, Furuhata A, Iwase H, Igarashi $\mathrm{M}$, et al. Low-intensity pulsed ultrasound (LIPUS) increases the articular cartilage type II collagen in a rat osteoarthritis model. J Orthop Res. 2010;28:361-9. doi:10.1002/jor.20995.

23. Naito K, Watari T, Furuhata A, Yomogida S, Sakamoto $\mathrm{K}$, Kurosawa $\mathrm{H}$, et al. Evaluation of the effect of glucosamine on an experimental rat osteoarthritis model. Life Sci. 2010;86(13-14):538-43. doi:10.1016/j.lfs.2010.02.015.

24. Hayami T, Pickarski M, Wesolowski GA, McLane J, Bone A, Destefano J, et al. The role of subchondral bone remodeling in osteoarthritis: reduction of cartilage degeneration and prevention of osteophyte formation by alendronate in the rat anterior cruciate ligament transection model. Arthritis Rheum. 2004;50(4):1193-206. doi:10.1002/art.20124.

25. Musumeci G, Castrogiovanni P, Trovato FM, Imbesi R, Giunta S, Szychlinska MA, et al. Physical activity ameliorates cartilage degeneration in a rat model of aging: a study on lubricin expression. Scand J Med Sci Sports. 2015;25(2):e222-30. doi: $10.1111 /$ sms. 12290 .

26. Appleton CT, Usmani SE, Pest MA, Pitelka V, Mort JS, Beier F. Reduction in disease progression by inhibition of transforming growth factor $\alpha$-CCL2 signaling in experimental posttraumatic osteoarthritis. Arthritis Rheumatol. 2015;67(10):2691-701. doi:10.1002/art.39255.

27. Ferrándiz ML, Terencio MC, Carceller MC, Ruhí R, Dalmau $\mathrm{P}$, Vergés J, et al. Effects of BIS076 in a model of osteoarthritis induced by anterior cruciate ligament transection in ovariectomised rats. BMC Musculoskelet Disord. 2015;16:92. doi: 10.1186/s12891-015-0547-9.

28. Yang JH, Kim JH, Lim DS, Oh KJ. Effect of combined sex hormone replacement on bone/cartilage turnover in a murine model of osteoarthritis. Clin Orthop Surg. 2012;4(3):234-41. doi:10.4055/cios.2012.4.3.234.

29. Orita S, Ishikawa T, Miyagi M, Ochiai N, Inoue G, Eguchi $\mathrm{Y}$, et al. Pain-related sensory innervation in monoiodoacetate-induced osteoarthritis in rat knees that gradually develops neuronal injury in addition to inflammatory pain. BMC Musculoskelet Disord. 2011;12:134. doi:10.1186/1471-247412-134.

30. Swearingen CA, Chambers MG, Lin C, Marimuthu J, Rito CJ, Carter QL, et al. A short-term pharmacodynamic model for monitoring aggrecanase activity: injection of monosodium iodoacetate (MIA) in rats and assessment of aggrecan neoepitope release in synovial fluid using novel ELISAs. Osteoarthritis Cartilage. 2010;18(9):1159-66. doi:10.1016/j. joca.2010.02.019.

31. Dufield DR, Nemirovskiy OV, Jennings MG, Tortorella MD, Malfait AM, Mathews WR. An immunoaffinity liquid chromatography-tandem mass spectrometry assay for detection of endogenous aggrecan fragments in biological fluids: 
use as a biomarker for aggrecanase activity and cartilage degradation. Anal Biochem. 2010;406:113-23. doi:10.1016/j. ab.2010.06.044.

32. Chockalingam PS, Sun W, Rivera-Bermudez MA, Zeng W, Dufield DR, Larsson S, et al. Elevated aggrecanase activity in a rat model of joint injury is attenuated by an aggrecanase specific inhibitor. Osteoarthritis Cartilage. 2011;19(3):31523. doi:10.1016/j.joca.2010.12.004.

33. Dhaneshwar S, Patil D. Chondromodulating chimeric prodrugs of diacetylrhein: synthesis and evaluation in monoiodoacetate-induced hyperalgesia. Med Chem. 2013;9(3):449-58.

34. Finn A, Angeby Moller K, Gustafsson C, Abdelmoaty S, Nordahl G, Ferm M, et al. Influence of model and matrix on cytokine profile in rat and human. Rheumatology. 2014;53(12):2297-305. doi:10.1093/rheumatology/keu281.

35. Braza-Boils A, Ferrandiz ML, Terencio MC, Alcaraz MJ. Analysis of early biochemical markers and regulation by tin protoporphyrin IX in a model of spontaneous osteoarthritis. Exp Gerontol. 2012;47(5):406-9. doi:10.1016/j. exger.2012.03.005.

36. Sarukawa J, Takahashi M, Doi M, Suzuki D, Nagano A. A longitudinal analysis of urinary biochemical markers and bone mineral density in STR/Ort mice as a model of spontaneous osteoarthritis. Arthritis Rheum. 2010;62(2):463-71. doi:10.1002/art.27202.

37. Ameye LG, Deberg M, Oliveira M, Labasse A, Aeschlimann JM, Henrotin Y. The chemical biomarkers C2C, Coll2-1, and Coll2-1NO2 provide complementary information on type II collagen catabolism in healthy and osteoarthritic mice. Arthritis Rheum. 2007;56:3336-46. doi:10.1002/art.22875.

38. Chia WT, Pan RY, Tseng FJ, Chen YW, Feng CK, Lee HS, et al. Experimental osteoarthritis induced by surgical realignment of the patella in BALB/c mice. J Bone Joint Surg Br. 2010;92(12):1710-6. doi:10.1302/0301-620x.92b12.23366.

39. Seifer DR, Furman BD, Guilak F, Olson SA, Brooks SC 3rd, Kraus VB. Novel synovial fluid recovery method allows for quantification of a marker of arthritis in mice. Osteoarthritis Cartilage. 2008;16(12):1532-8. doi:10.1016/j. joca.2008.04.013.

40. Porta CL, Andreea Bura S, Llorente-Onaindia J, Pastor A, Navarrete F, García-Gutierrez MS, et al. Role of the endocannabinoid system in the emotional manifestations of osteoarthritis pain. Pain. 2015;156(10):2001-12. doi:10.1097/j. pain.0000000000000260.

41. Kyostio-Moore S, Nambiar B, Hutto E, Ewing PJ, Piraino S, Berthelette P, et al. STR/ort mice, a model for spontaneous osteoarthritis, exhibit elevated levels of both local and systemic inflammatory markers. Comp Med. 2011;61(4):346-55.

42. Lewis JS, Hembree WC, Furman BD, Tippets L, Cattel $\mathrm{D}$, Huebner JL, et al. Acute joint pathology and synovial inflammation is associated with increased intra-articular fracture severity in the mouse knee. Osteoarthritis Cartilage. 2011;19(7):864-73. doi:10.1016/j.joca.2011.04.011.

43. Watari T, Naito K, Sakamoto K, Kurosawa H, Nagaoka I, Kaneko K. Evaluation of the effect of oxidative stress on articular cartilage in spontaneously osteoarthritic STR/ OrtCrlj mice by measuring the biomarkers for oxidative stress and type II collagen degradation/synthesis. Exp Ther Med. 2011;2(2):245-50. doi:10.3892/etm.2011.196.
44. Horcajada MN, Sanchez C, Membrez Scalfo F, Drion P, Comblain F, Taralla S, et al. Oleuropein or rutin consumption decreases the spontaneous development of osteoarthritis in the Hartley guinea pig. Osteoarthritis Cartilage. 2015;23(1):94102. doi:10.1016/j.joca.2014.08.016.

45. Huebner JL, Hanes MA, Beekman B, TeKoppele JM, Kraus VB. A comparative analysis of bone and cartilage metabolism in two strains of guinea-pig with varying degrees of naturally occurring osteoarthritis. Osteoarthritis Cartilage. 2002;10(10):758-67. doi:10.1053/joca.2002.0821.

46. Huebner JL, Kraus VB. Assessment of the utility of biomarkers of osteoarthritis in the guinea pig. Osteoarthritis Cartilage. 2006;14(9):923-30. doi:10.1016/j.joca.2006.03.007.

47. Huebner JL, Williams JM, Deberg M, Henrotin Y, Kraus VB. Collagen fibril disruption occurs early in primary guinea pig knee osteoarthritis. Osteoarthritis Cartilage. 2010;18(3):397405. doi:10.1016/j.joca.2009.09.011.

48. Wei L, Fleming BC, Sun X, Teeple E, Wu W, Jay GD, et al. Comparison of differential biomarkers of osteoarthritis with and without posttraumatic injury in the Hartley guinea pig model. J Orthop Res. 2010;28(7):900-6. doi:10.1002/ jor. 21093 .

49. Huebner JL, Seifer DR, Kraus VB. A longitudinal analysis of serum cytokines in the Hartley guinea pig model of osteoarthritis. Osteoarthritis Cartilage. 2007;15(3):354-6. doi:10.1016/j.joca.2006.10.014.

50. Huebner JL, Johnson KA, Kraus VB, Terkeltaub RA. Transglutaminase 2 is a marker of chondrocyte hypertrophy and osteoarthritis severity in the Hartley guinea pig model of knee OA. Osteoarthritis Cartilage. 2009;17(8):1056-64. doi:10.1016/j.joca.2009.02.015.

51. Knott L, Avery NC, Hollander AP, Tarlton JF. Regulation of osteoarthritis by omega-3 (n-3) polyunsaturated fatty acids in a naturally occurring model of disease. Osteoarthritis Cartilage. 2011;19(9):1150-7. doi:10.1016/j.joca.2011.06.005.

52. McDougall JJ, Schuelert N, Bowyer J. Cathepsin K inhibition reduces CTXII levels and joint pain in the guinea pig model of spontaneous osteoarthritis. Osteoarthritis Cartilage. 2010;18(10):1355-7. doi:10.1016/j.joca.2010.07.014.

53. Lamers RJ, DeGroot J, Spies-Faber EJ, Jellema RH, Kraus $\mathrm{VB}$, Verzijl N, et al. Identification of disease- and nutrientrelated metabolic fingerprints in osteoarthritic guinea pigs. $\mathrm{J}$ Nutr. 2003;133(6):1776-80.

54. Matyas JR, Atley L, Ionescu M, Eyre DR, Poole AR. Analysis of cartilage biomarkers in the early phases of canine experimental osteoarthritis. Arthritis Rheum. 2004;50(2):543-52. doi:10.1002/art.20027.

55. Henrotin Y, Martel-Pelletier J, Msika P, Guillou GB, Deberg M. Usefulness of specific OA biomarkers, Coll2-1 and Coll2$1 \mathrm{NO} 2$, in the anterior cruciate ligament OA canine model. Osteoarthritis Cartilage. 2012;20(7):787-90. doi:10.1016/j. joca.2012.03.016.

56. Thonar EJ, Masuda K, Lenz ME, Hauselmann HJ, Kuettner KE, Manicourt DH. Serum markers of systemic disease processes in osteoarthritis. J Rheumatol Suppl. 1995;43:68-70.

57. Chockalingam PS, Glasson SS, Lohmander LS. Tenascin-C levels in synovial fluid are elevated after injury to the human and canine joint and correlate with markers of inflammation and matrix degradation. Osteoarthritis Cartilage. 2013;21(2):339-45. doi:10.1016/jjoca.2012.10.016. 
58. Dearmin MG, Trumble TN, Garcia A, Chambers JN, Budsberg SC. Chondroprotective effects of zoledronic acid on articular cartilage in dogs with experimentally induced osteoarthritis. Am J Vet Res. 2014;75(4):329-37. doi:10.2460/ajvr.75.4.329.

59. Connor JR, LePage C, Swift BA, Yamashita D, Bendele AM, Maul D, et al. Protective effects of a cathepsin $\mathrm{K}$ inhibitor, SB-553484, in the canine partial medial meniscectomy model of osteoarthritis. Osteoarthritis Cartilage. 2009;17(9):123643. doi:10.1016/j.joca.2009.03.015.

60. Settle S, Vickery L, Nemirovskiy O, Vidmar T, Bendele A, Messing D, et al. Cartilage degradation biomarkers predict efficacy of a novel, highly selective matrix metalloproteinase 13 inhibitor in a dog model of osteoarthritis: confirmation by multivariate analysis that modulation of type II collagen and aggrecan degradation peptides parallels pathologic changes. Arthritis Rheum. 2010;62(10):3006-15. doi:10.1002/art.27596.

61. Rhouma M, de Oliveira El, Warrak A, Troncy E, Beaudry F, Chorfi Y. Anti-inflammatory response of dietary vitamin $\mathrm{E}$ and its effects on pain and joint structures during early stages of surgically induced osteoarthritis in dogs. Can J Vet Res. 2013;77(3):191-8.

62. Alam MR, Ji JR, Kim MS, Kim NS. Biomarkers for identifying the early phases of osteoarthritis secondary to medial patellar luxation in dogs. J Vet Sci. 2011;12(3):273. doi:10.4142/ jvs.2011.12.3.273.

63. Hegemann N, Kohn B, Brunnberg L, Schmidt MF. Biomarkers of joint tissue metabolism in canine osteoarthritic and arthritic joint disorders. Osteoarthritis Cartilage. 2002;10(9):714-21. doi:10.1053/joca.2002.0820.

64. Pelletier JP, Lajeunesse D, Jovanovic DV, Lascau-Coman V, Jolicoeur FC, Hilal G, et al. Carprofen simultaneously reduces progression of morphological changes in cartilage and subchondral bone in experimental dog osteoarthritis. J Rheumatol. 2000;27(12):2893-902.

65. Mastbergen SC, Marijnissen AC, Vianen ME, Zoer B, van Roermund PM, Bijlsma JW, et al. Inhibition of COX-2 by celecoxib in the canine groove model of osteoarthritis. Rheumatology. 2006;45(4):405-13. doi:10.1093/rheumatology/kei187.

66. Gharbi M, Sanchez C, Mazzucchelli G, De Pauw E, Henrotin Y. Identification of differential pattern of protein expression in canine osteoarthritis serum after anterior cruciate ligament transection: a proteomic analysis. Vet J. 2013;197(3):848-53. doi:10.1016/j.tvj1.2013.05.037.

67. Lindhorst E, Vail TP, Guilak F, Wang H, Setton LA, Vilim $\mathrm{V}$, et al. Longitudinal characterization of synovial fluid biomarkers in the canine meniscectomy model of osteoarthritis. J Orthop Res. 2000;18(2):269-80. doi:10.1002/ jor. 1100180216 .

68. van Vulpen LF, van Meegeren ME, Roosendaal G, Jansen NW, van Laar JM, Schutgens RE, et al. Biochemical markers of joint tissue damage increase shortly after a joint bleed; an explorative human and canine in vivo study. Osteoarthritis Cartilage. 2015;23(1):63-9. doi:10.1016/j.joca.2014.09.008.

69. Manicourt DH, Altman RD, Williams JM, Devogelaer JP, Druetz-Van Egeren A, Lenz ME, et al. Treatment with calcitonin suppresses the responses of bone, cartilage, and synovium in the early stages of canine experimental osteoarthritis and significantly reduces the severity of the cartilage lesions.
Arthritis Rheum. 1999;42(6):1159-67. doi:10.1002/15290131(199906)42:6<1159::aid-anr12>3.0.co;2-q.

70. Kawcak CE, Frisbie DD, Werpy NM, Park RD, McIlwraith CW. Effects of exercise vs experimental osteoarthritis on imaging outcomes. Osteoarthritis Cartilage. 2008;16(12):1519-25. doi:10.1016/jjoca.2008.04.015.

71. Verwilghen DR, Enzerink E, Martens A, Franck T, Balligand $\mathrm{M}$, Henrotin $\mathrm{Y}$, et al. Relationship between arthroscopic joint evaluation and the levels of Coll2-1, Coll2-1NO(2), and myeloperoxidase in the blood and synovial fluid of horses affected with osteochondrosis of the tarsocrural joint. Osteoarthritis Cartilage. 2011;19(11):1323-9. doi:10.1016/j. joca.2011.08.002.

72. Verwilghen DR, Martens A, Busschers E, Franck T, Deberg $\mathrm{M}$, Henrotin Y, et al. Coll2-1, Coll2-1NO2 and myeloperoxidase concentrations in the synovial fluid of equine tarsocrural joints affected with osteochondrosis. Vet Res Commun. 2011;35(7):401-8. doi:10.1007/s11259-011-9487-5.

73. Billinghurst R. Significant exercise-related changes in the serum levels of two biomarkers of collagen metabolism in young horses. Osteoarthritis Cartilage. 2003;11(10):760-9. doi:10.1016/s1063-4584(03)00152-3.

74. Frisbie DD, Al-Sobayil F, Billinghurst RC, Kawcak CE, McIlwraith CW. Changes in synovial fluid and serum biomarkers with exercise and early osteoarthritis in horses. Osteoarthritis Cartilage. 2008;16(10):1196-204. doi:10.1016/ jjoca.2008.03.008.

75. Fuller CJ, Barr AR, Sharif M, Dieppe PA. Cross-sectional comparison of synovial fluid biochemical markers in equine osteoarthritis and the correlation of these markers with articular cartilage damage. Osteoarthritis Cartilage. 2001;9(1):4955. doi:10.1053/joca.2000.0349.

76. Maninchedda U, Lepage OM, Gangl M, Hilairet S, Remandet $\mathrm{B}$, Meot F, et al. Development of an equine groove model to induce metacarpophalangeal osteoarthritis: a pilot study on 6 horses. PLoS One. 2015;10(2):e0115089. doi:10.1371/journal.pone.0115089.

77. Maher AD, Coles C, White J, Bateman JF, Fuller ES, Burkhardt D, et al. ${ }^{1} \mathrm{H}$ NMR spectroscopy of serum reveals unique metabolic fingerprints associated with subtypes of surgically induced osteoarthritis in sheep. J Proteome Res. 2012;11(8):4261-8. doi:10.1021/pr300368h.

78. Miyamoto K, Ishimaru J, Kurita K, Goss AN. Synovial matrix metalloproteinase-2 in different stages of sheep temporomandibular joint osteoarthrosis. J Oral Maxillofac Surg. 2002;60(1):66-72.

79. Mickiewicz B, Heard BJ, Chau JK, Chung M, Hart DA, Shrive $\mathrm{NG}$, et al. Metabolic profiling of synovial fluid in a unilateral ovine model of anterior cruciate ligament reconstruction of the knee suggests biomarkers for early osteoarthritis. J Orthop Res. 2015;33(1):71-7. doi:10.1002/jor.22743.

80. Kraus VB, Huebner JL, Fink C, King JB, Brown S, Vail TP, et al. Urea as a passive transport marker for arthritis biomarker studies. Arthritis Rheum. 2002;46(2):420-7.

81. Kraus VB, Burnett B, Coindreau J, Cottrell S, Eyre D, Gendreau M, et al. Application of biomarkers in the development of drugs intended for the treatment of osteoarthritis. Osteoarthritis Cartilage. 2011;19(5):515-42. doi:10.1016/j. joca.2010.08.019. 